INTERNATIONAL JOURNAL FOR NUMERICAL METHODS IN FLUIDS

Int. J. Numer. Meth. Fluids (2009)

Published online in Wiley InterScience (www.interscience.wiley.com). DOI: 10.1002/fld.2040

\title{
Global flow instability in a lid-driven cavity
}

\author{
V. B. L. Boppana ${ }^{1}$ and J. S. B. Gajjar ${ }^{2, *, \dagger}$ \\ ${ }^{1}$ School of Engineering Sciences, University of Southampton, Highfield, Southampton SO17 1BJ, U.K. \\ ${ }^{2}$ School of Mathematics, The University of Manchester, Alan Turing Building, Manchester M13 9PL, U.K.
}

\begin{abstract}
SUMMARY
The stability of flow in a lid-driven cavity is investigated using an accurate numerical technique based on a hybrid scheme with spectral collocation and high-order finite differences. A global stability analysis is carried out and critical parameters are identified for various aspect ratios. It is found that while there is reasonable agreement with the literature for the critical parameters leading to loss of stability for the square cavity, there are significant discrepancies for cavities of aspect ratios 1.5 and 2. Simulations of the linearized unsteady equations confirm the results from the global stability analysis for aspect ratios $A=1,1.5$ and $A=2$. Copyright (C) 2009 John Wiley \& Sons, Ltd.
\end{abstract}

Received 3 July 2008; Revised 14 January 2009; Accepted 6 February 2009

KEY WORDS: Hopf bifurcation; lid-driven cavity; global stability; aspect ratio; Navier-Stokes

\section{INTRODUCTION}

The flow in a lid-driven cavity (LDC) has been extensively used as a test case for the development of numerical algorithms for the Navier-Stokes equations and there are many papers Burgraff [1], Ghia et al. [2], Schrieber and Keller [3] that describe the various flow features. In [2] results for a wide range of Reynolds numbers up to 10000 are presented. In the singular LDC problem, the one studied here, there is a discontinuity in the velocity boundary conditions at the corners where the lid moves, and in more recent work Botella and Peyret [4], Auteri et al. [5] have attempted to obtain accurate solutions to the steady flow problem by incorporating the nature of the singularity at the corners into their numerical algorithms. One of the interests from the theoretical perspective is to obtain an insight into solution properties of the steady flow in the limit so that the Reynolds numbers become large. Solutions of the steady problem for Reynolds numbers up to 20000 have been obtained by Gajjar and Azzam [6], Azzam [7], Erturk et al. [8] and show the core flow tending to a Prandtl-Batchelor type of flow, see Batchelor [9], with closed streamlines and with the core vorticity tending to a uniform value.

\footnotetext{
${ }^{*}$ Correspondence to: J. S. B. Gajjar, School of Mathematics, The University of Manchester, Alan Turing Building, Manchester M13 9PL, U.K.

†E-mail: j.gajjar@manchester.ac.uk
} 
The focus of the current work is to investigate the linear stability of the two-dimensional LDC flow, for various aspect (depth/width) ratios, using an accurate numerical method that employs Chebyshev collocation in one direction together with high-order finite differences in the other direction. The same numerical technique is employed by Azzam [7] for the cavity flow, by Gajjar and Azzam [10] to compute the steady flow past an array of cylinders for large Reynolds numbers and by Korolev et al. [11] for the supersonic flow past a compression ramp for large ramp angles. One of the reasons why the technique has been successfully used in a wide range of problems cited above is that the Newton linearization of the nonlinear equations leads to a linear system that has a block-pentadiagonal structure, and this is solved directly using an efficient solver.

The stability of the LDC flow has been investigated by a number of authors, see Gustafson and Halasi [12,13], Goodrich et al. [14]. They find from the simulations of the full time-dependent Navier-Stokes equations that the steady flow loses stability via a Hopf bifurcation. To obtain accurate values of the critical parameters for loss of stability via simulations of the unsteady equations is in general a difficult task and computationally expensive. In recent work Auteri et al. [15] have taken account of the corner singularities in the LDC problem and via simulations managed to narrow the range of values of the critical Reynolds number when a Hopf bifurcation takes place. Poliashenko and Aidun [16] have reviewed a number of different techniques that can also be used to study the stability of the steady state and to compute bifurcations. In [16, 17], among others, the two-dimensional eigenvalue problem is solved numerically and the critical Reynolds numbers and frequencies from the eigenvalue analysis are compared with simulations of the unsteady equations.

One of the aims of the current work is to obtain accurate value of the critical parameters for loss of stability for cavity aspect ratios of $1,1.5$ and 2. A survey of the literature suggests that the range of critical Reynolds numbers identified when the flow becomes unstable for the square cavity is quite varied, from 7704 in [18] to 8031.93 in [19]. For aspect ratio of 1.5 there is a huge discrepancy between the results of [16] and [20]. Likewise there are not many results for the cavity of aspect ratio 2 .

In the present study we have used a robust and accurate numerical technique to first obtain the steady flow. The same technique is used to investigate the stability of the flow by solving the generalized two-dimensional eigenvalue problem obtained from the linearized unsteady equations. The stability results are confirmed also via simulations of the linearized unsteady equations for the aspect ratios studied.

In Section 2 we consider the governing equations. The details of the stability analysis and numerical techniques used are discussed in Sections 3 and 4. In Section 5 we present our numerical results and compare them with the previous work.

\section{GOVERNING EQUATIONS}

The non-dimensional equations that describe the two-dimensional, incompressible and unsteady fluid flow in a LDC in terms of a stream function $(\psi)$ and vorticity $(\omega)$ are

$$
\begin{aligned}
\nabla^{2} \psi & =-\omega \quad \text { and } \\
\omega_{t}+\psi_{y} \omega_{x}-\psi_{x} \omega_{y} & =\frac{1}{R e} \nabla^{2} \omega
\end{aligned}
$$




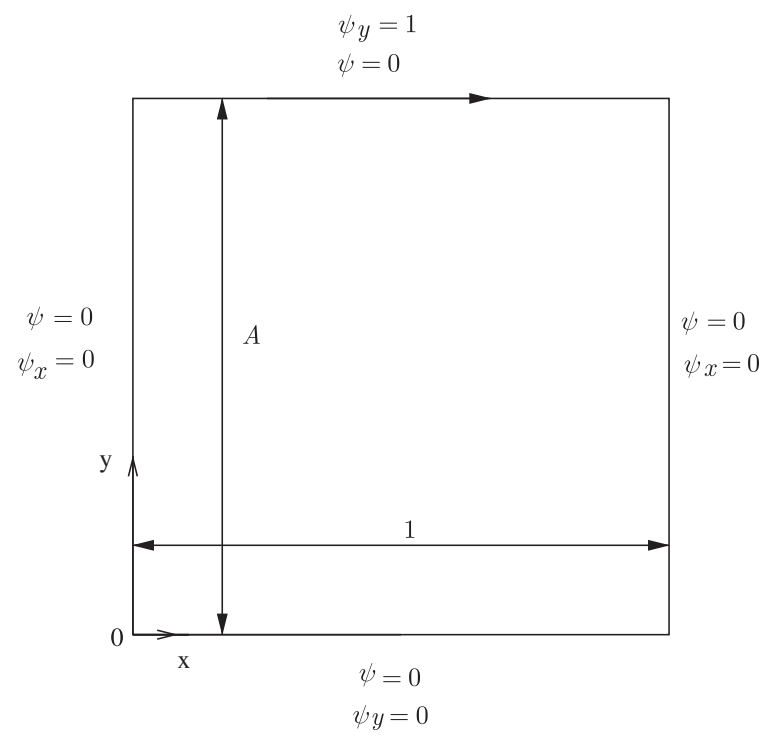

Figure 1 . Sketch of the lid-driven cavity with boundary conditions.

Here $R e$ is the Reynolds number defined as $U w / v$, where $v$ is the kinematic viscosity of the fluid, $U$ is the velocity of the lid, and $w$ is the width of the cavity that are used to non-dimensionalize the velocity and length-scale variables, respectively. The boundary conditions (see Figure 1) are given by

$$
\begin{aligned}
& \psi=0 \quad \text { and } \quad \psi_{x}=0 \quad \text { for } x=0,0 \leqslant y \leqslant A \\
& \psi=0 \text { and } \psi_{x}=0 \text { for } x=1,0 \leqslant y \leqslant A \\
& \psi=0 \text { and } \psi_{y}=0 \text { for } y=0,0 \leqslant x \leqslant 1 \\
& \psi=0 \text { and } \psi_{y}=1 \text { for } y=A, 0 \leqslant x \leqslant 1
\end{aligned}
$$

where $A$ is the aspect ratio of the cavity and is defined as

$$
A=\frac{\text { depth of the cavity }}{\text { width of the cavity }}=\frac{d}{w}
$$

In the present study, three different aspect ratios are considered, $A=1.0,1.5$ and 2.0.

\section{HYDRODYNAMIC STABILITY ANALYSIS}

To determine the stability we used a normal mode analysis according to which the total flow $(\psi(x, y, t)$ and $\omega(x, y, t))$ are expressed as

$$
\begin{aligned}
& \psi(x, y, t)=\bar{\psi}(x, y)+\delta \tilde{\psi}(x, y) \mathrm{e}^{\lambda t} \quad \text { and } \\
& \omega(x, y, t)=\bar{\omega}(x, y)+\delta \widetilde{\omega}(x, y) \mathrm{e}^{\lambda t}
\end{aligned}
$$


where the barred quantities denote the steady flow and the terms multiplied by $\delta$ the perturbation. Substituting the above form into Equations (1) and (2), assuming that $\delta$ is small and collecting the like order terms will give rise to a set of equations for the steady flow and the linear stability equations.

Steady equations and their boundary conditions:

$$
\begin{gathered}
\nabla^{2} \bar{\psi}=-\bar{\omega} \\
\bar{\psi}_{y} \bar{\omega}_{x}-\bar{\psi}_{x} \bar{\omega}_{y}=\frac{1}{\operatorname{Re}} \nabla^{2} \bar{\omega} \\
\bar{\psi}=0 \quad \text { and } \quad \bar{\psi}_{x}=0 \quad \text { for } x=0, \quad 0 \leqslant y \leqslant A \\
\bar{\psi}=0 \quad \text { and } \quad \bar{\psi}_{x}=0 \quad \text { for } x=1, \quad 0 \leqslant y \leqslant A \\
\bar{\psi}=0 \quad \text { and } \quad \bar{\psi}_{y}=0 \text { for } y=0, \quad 0 \leqslant x \leqslant 1 \\
\bar{\psi}=0 \quad \text { and } \quad \bar{\psi}_{y}=1 \quad \text { for } y=A, \quad 0 \leqslant x \leqslant 1
\end{gathered}
$$

Stability equations and their boundary conditions:

$$
\begin{gathered}
\nabla^{2} \widetilde{\psi}=-\widetilde{\omega} \\
\lambda \widetilde{\omega}+\bar{\psi}_{y} \widetilde{\omega}_{x}-\bar{\psi}_{x} \widetilde{\omega}_{y}+\widetilde{\psi}_{y} \bar{\omega}_{x}-\widetilde{\psi}_{x} \bar{\omega}_{y}=\frac{1}{R e} \nabla^{2} \widetilde{\omega} \\
\widetilde{\psi}=0 \quad \text { and } \quad \widetilde{\psi}_{x}=0 \quad \text { for } x=0, \quad 0 \leqslant y \leqslant A \\
\widetilde{\psi}=0 \quad \text { and } \quad \widetilde{\psi}_{x}=0 \quad \text { for } x=1, \quad 0 \leqslant y \leqslant A \\
\widetilde{\psi}=0 \quad \text { and } \quad \widetilde{\psi}_{y}=0 \quad \text { for } y=0,0 \leqslant x \leqslant 1 \\
\widetilde{\psi}=0 \quad \text { and } \quad \widetilde{\psi}_{y}=0 \text { for } y=A, \quad 0 \leqslant x \leqslant 1
\end{gathered}
$$

Initially, the steady Equations (4) are solved for $\bar{\psi}$ and $\bar{\omega}$. The obtained steady solution is then substituted into (5), which is a two-dimensional partial differential eigenvalue problem. After discretization this leads to a generalized eigenvalue problem that is solved to find $\widetilde{\psi}, \widetilde{\omega}$ and $\lambda$ for different $R e$. In general, the eigenvalues $\lambda$ can be real $\left(\lambda=\lambda_{r}\right)$ or complex $\left(\lambda=\lambda_{r}+\lambda_{i}\right)$. The stability of the flow depends on the sign of the largest value of $\lambda_{r}$. For the flow in a LDC, at one particular value of $R e$, a pair of complex conjugate values of $\lambda$ crosses the imaginary axis. This leads the basic flow to lose stability and a one parameter family of periodic solution bifurcates from the stationary solution. Such a bifurcation is called a Hopf bifurcation. The numerical technique used to determine the basic flow and its stability is explained in the next section. 


\section{GLOBAL FLOW INSTABILITY IN AN LDC}

\section{NUMERICAL METHOD}

Fourth-order central differencing in the $x$-direction and Chebyshev collocation in the $y$-direction are used for discretizing equations (4) and (5). Accordingly, the first and second derivatives in the $x$-direction are differenced as

$$
\begin{aligned}
\left(\Upsilon_{x}\right)_{i, j} & =\frac{1}{12 h}\left(\Upsilon_{i-2, j}-8 \Upsilon_{i-1, j}+8 \Upsilon_{i+1, j}-\Upsilon_{i+2, j}\right) \quad \text { and } \\
\left(\Upsilon_{x x}\right)_{i, j} & =\frac{1}{12 h^{2}}\left(-\Upsilon_{i-2, j}+16 \Upsilon_{i-1, j}-30 \Upsilon_{i, j}+16 \Upsilon_{i+1, j}-\Upsilon_{i+2, j}\right)
\end{aligned}
$$

where suffices $i, j$ refer to points $x_{i}, y_{j}$. Here $x_{i}=x_{a}+\mathrm{i} h, \quad i=0,1, \ldots, m$ and $h=\left(x_{b}-x_{a}\right) / m$ (where $x_{a}=0$ and $x_{b}=1$ ). In the $y$-direction, the physical domain of $y$ is mapped to Chebyshev space i.e. $y \in[0, A] \rightarrow z \in[-1,1]$ where

$$
z_{j}=\cos \left(\frac{j \pi}{n}\right), \quad j=0,1, \ldots, n \quad \text { and } \quad y_{j}=A\left(\frac{z_{j}+1}{2}\right)
$$

Hence the first and second derivatives in the $y$-direction are given by

$$
\begin{aligned}
\left(\Upsilon_{y}\right)_{i, j} & =\sum_{k=0}^{n} d 1_{j, k} \Upsilon_{i, k} \quad \text { and } \\
\left(\Upsilon_{y y}\right)_{i, j} & =\sum_{k=0}^{n} d 2_{j, k} \Upsilon_{i, k}
\end{aligned}
$$

Here $d 1_{j, k}$ and $d 2_{j, k}$ are the elements of the Chebyshev collocation differentiation matrix of $\mathscr{D}$ and $\mathscr{D}^{2}$, respectively, (for the details of the matrix $\mathscr{D}$, refer Canuto et al. [21]) and $\Upsilon$ can be $\bar{\psi}, \bar{\omega}, \widetilde{\psi}$ and $\widetilde{\omega}$. Let $M=m+1$ and $N=n+1$ be the total number of grid points in the $x$-and $y$-directions, respectively.

Substituting Equations (6) and (7) in basic flow equations (4) gives rise to a set of discrete equations which are nonlinear. These are linearized using a Newton-Raphson technique. For this purpose, let $\bar{\psi}_{i, j}=\Psi_{i, j}+H_{i, j}$ and $\bar{\omega}_{i, j}=\Omega_{i, j}+G_{i, j}$, where $\Psi_{i, j}, \Omega_{i, j}$ are some initial guesses and $H_{i, j}, G_{i, j}$ are corresponding correction factors such that $\left|H_{i, j}\right|,\left|G_{i, j}\right| \ll 1$. Thus the linearized steady equations, after ignoring the smaller order nonlinear terms and collecting the like terms will be of the form

$$
\overline{\mathbf{A}}^{(p)} \boldsymbol{\Phi}_{p-2}+\overline{\mathbf{B}}^{(p)} \boldsymbol{\Phi}_{p-1}+\overline{\mathbf{C}}^{(p)} \boldsymbol{\Phi}_{p}+\overline{\mathbf{D}}^{(p)} \boldsymbol{\Phi}_{p+1}+\overline{\mathbf{E}}^{(p)} \boldsymbol{\Phi}_{p+2}=\mathbf{R}^{(p)}, \quad 1 \leqslant p \leqslant M
$$

where the matrices $\overline{\mathbf{A}}$ to $\overline{\mathbf{E}}$ are of size $2 N \times 2 N$ and they arise due to the enforcement of equations at the Chebyshev collocation nodes in the $y$-direction. $\boldsymbol{\Phi}_{p}$ is a vector of correction factors of stream function $\left(H_{p}\right)$ and vorticity $\left(G_{p}\right)$ at each node in the $x$-direction i.e.

$$
\boldsymbol{\Phi}_{p}=\left(\begin{array}{l}
\underline{H}_{p} \\
\underline{G}_{p}
\end{array}\right)=\left(H_{p, 0}, \ldots, H_{p, n}, G_{p, 0}, \ldots, G_{p, n}\right)^{\mathrm{T}}
$$

A block penta-diagonal structure arises as a result of discretizing the equations with fourth-order central differences in the $x$-direction. The linear system (8), when written in matrix form, results in

$$
\overline{\mathbf{L}} \boldsymbol{\Phi}=\overline{\mathbf{R}}
$$


with $\boldsymbol{\Phi}=\left(\boldsymbol{\Phi}_{1}, \ldots, \boldsymbol{\Phi}_{M}\right)^{\mathrm{T}}$. Equation (9) is solved for $\boldsymbol{\Phi}$ using a direct solver that exploits the sparsity of the block penta-diagonal structure of the matrix $\overline{\mathbf{L}}$.

The obtained stationary solution is used in Equation (5) that after discretization and collecting the like terms gives rise to

$$
\widetilde{\mathbf{A}}^{(p)} \boldsymbol{\Theta}_{p-2}+\widetilde{\mathbf{B}}^{(p)} \boldsymbol{\Theta}_{p-1}+\widetilde{\mathbf{C}}^{(p)} \boldsymbol{\Theta}_{p}+\widetilde{\mathbf{D}}^{(p)} \boldsymbol{\Theta}_{p+1}+\widetilde{\mathbf{E}}^{(p)} \boldsymbol{\Theta}_{p+2}=\lambda \widetilde{\mathbf{R}}^{(p)} \boldsymbol{\Theta}_{p}, \quad 1 \leqslant p \leqslant M
$$

where $\boldsymbol{\Theta}_{k}$ is a vector similar to $\boldsymbol{\Phi}_{k}$ containing the perturbation stream function and vorticity. Matrices $\widetilde{\mathbf{A}}^{(p)}$ to $\widetilde{\mathbf{E}}^{(p)}$ are similar to $\overline{\mathbf{A}}^{(p)}$ and $\overline{\mathbf{E}}^{(p)}$ except that $\Psi$ and $\Omega$ in them have to be replaced by $\bar{\psi}$ and $\bar{\omega}$. Equation (10) can be written in the form of a generalized eigenvalue problem given by

$$
\widetilde{\mathbf{L}} \boldsymbol{\Theta}=\lambda \widetilde{\mathbf{R}} \boldsymbol{\Theta}
$$

where $\widetilde{\mathbf{R}}$ is a diagonal matrix and $\boldsymbol{\Theta}=\left[\boldsymbol{\Theta}_{\mathbf{1}}, \boldsymbol{\Theta}_{\mathbf{2}}, \ldots, \boldsymbol{\Theta}_{\mathbf{M}-\mathbf{1}}, \boldsymbol{\Theta}_{\mathbf{M}}\right]^{\mathrm{T}}$. Equation (11) is solved for eigenvalues $\lambda$ and eigenvectors $\Theta$ using ARPACK [22].

\subsection{Implementation of boundary conditions for vorticity}

We used an integral constraint method to enforce the no-slip boundary conditions. Accordingly, Equation (4a) is integrated with respect to $y$, which then gives

$$
\left[\bar{\psi}_{y}\right]_{0}^{y}+\int_{0}^{y}\left(\frac{\partial^{2} \bar{\psi}}{\partial x^{2}}+\bar{\omega}\right) \mathrm{d} y=0
$$

Evaluating this at $y=A$ and using the conditions of $\bar{\psi}_{y}$ given in Equation (4c), we get

$$
1+\int_{0}^{A}\left(\frac{\partial^{2} \bar{\psi}}{\partial x^{2}}+\bar{\omega}\right) \mathrm{d} y=0
$$

This gives the vorticity boundary condition at $y=0$. For $y=A$ and $0 \leqslant x \leqslant 1$, the boundary condition for vorticity is obtained by double integrating Equation (4a) with respect to $y$ and then using the conditions, $\bar{\psi}(y=A)=\bar{\psi}(y=0)=\bar{\psi}_{y}(y=0)=0$, which then gives

$$
\int_{0}^{A}\left[\int_{0}^{y}\left(\frac{\partial^{2} \bar{\psi}}{\partial x^{2}}+\bar{\omega}\right) \mathrm{d} y\right] \mathrm{d} y=0
$$

At $x=0$ and 1, Equation (4a) is used with $\bar{\psi}_{x}=0$ to eliminate the points outside the domain. The boundary conditions are handled in a similar manner for stability equations.

\subsection{Validation of numerical method}

In order to validate the techniques described above, extensive tests were carried out with benchmark problems. For the steady version of the code, we solved the steady LDC problem given by Equations (4) and compared our results with Ghia et al. [2]. For example, in Figure 2 we show contours of the streamlines and vorticity for $R e=7500$, for a grid size with $N=97, M=257$, and in Figure 3 the centerline plots of the velocity are compared with the data from [2]. Both are in excellent agreement. Further grid size checks and other more extensive comparisons of the data 

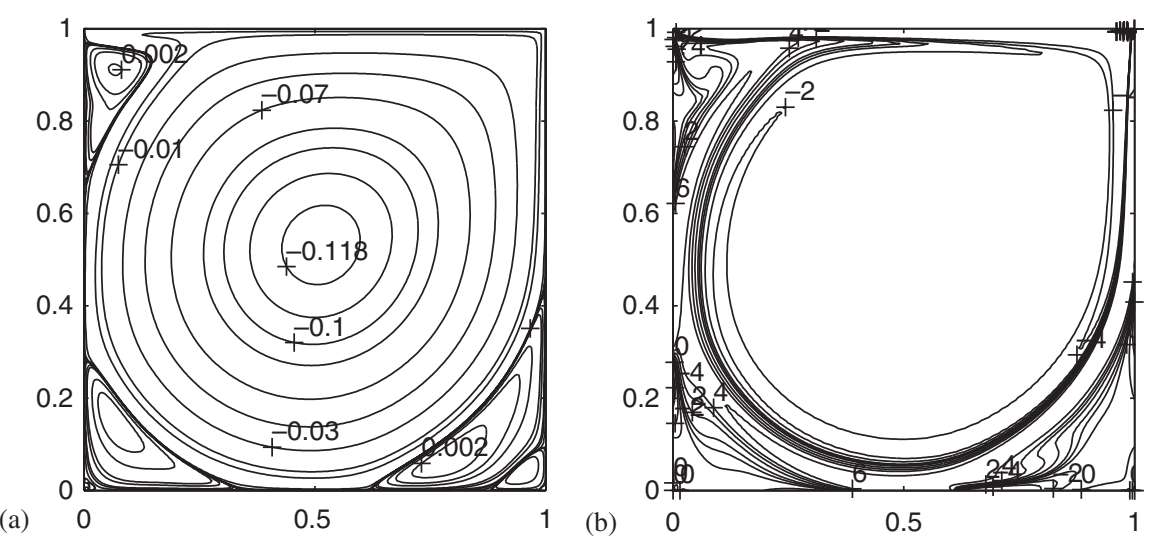

Figure 2. Contours of the (a) stream function and (b) vorticity for $R e=7500$ for the LDC flow. Contour levels shown are as in [2].

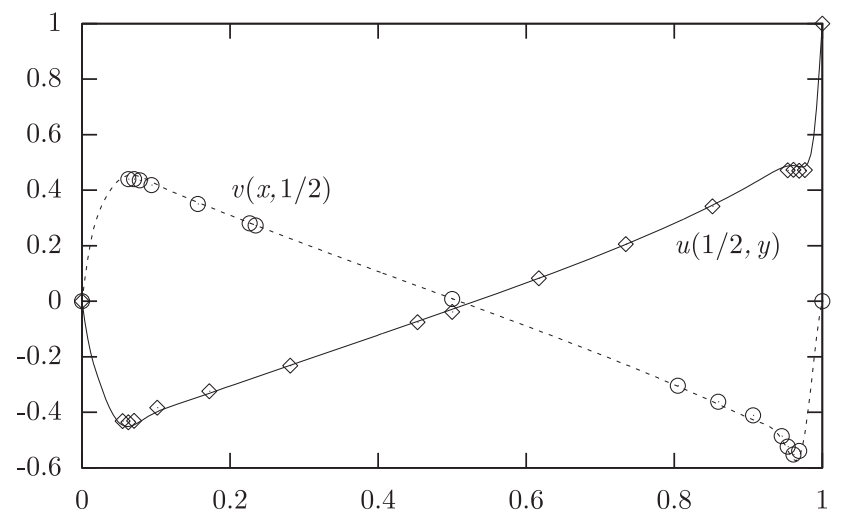

Figure 3. A comparison of the centerline velocities $u\left(\frac{1}{2}, y\right), v\left(x, \frac{1}{2}\right)$ (solid lines) for $R e=7500$ with data (symbols) from [2].

for the LDC flow, for a wide range of Reynolds numbers, generated using these techniques for both second- and fourth-order differencing in the $x$-direction are given in [7].

To test the eigenvalue solver using the discretization techniques described above, we again solved a number of different benchmark problems. In particular, the method was successfully used to obtain eigenvalues for two fourth-order eigenvalue problems, which are the biharmonic eigenvalue problem and the buckling plate problem discussed by Bjørstad and Tjøstheim in [23] and which are described below.

The eigenvalue problem for the biharmonic operator is

$$
\omega=\nabla^{2} \psi, \quad \nabla^{2} \omega=\lambda \psi, 0<x, y<1, \psi=\frac{\partial \psi}{\partial n}=0 \text { on the boundary }
$$


Table I. Comparison of the smallest eigenvalue obtained by Bjørstad and Tjøstheim [23] in this study.

\begin{tabular}{lrcc}
\hline & $M$ & $N=13$ & $N=26$ \\
\hline Biharmonic eigenvalue problem & 51 & 1294.93636 & 1294.93537 \\
Present study & 101 & 1294.93500 & 1294.93400 \\
Bjørstad and Tjøstheim [23] & \multicolumn{3}{c}{1294.93398} \\
Buckling plate eigenvalue problem & 51 & 52.3443114 & 52.3443114 \\
Present study & 101 & 52.3446434 & 52.344644 \\
Bjørstad and Tjøstheim [23] & \multicolumn{3}{c}{52.3446913} \\
\hline
\end{tabular}
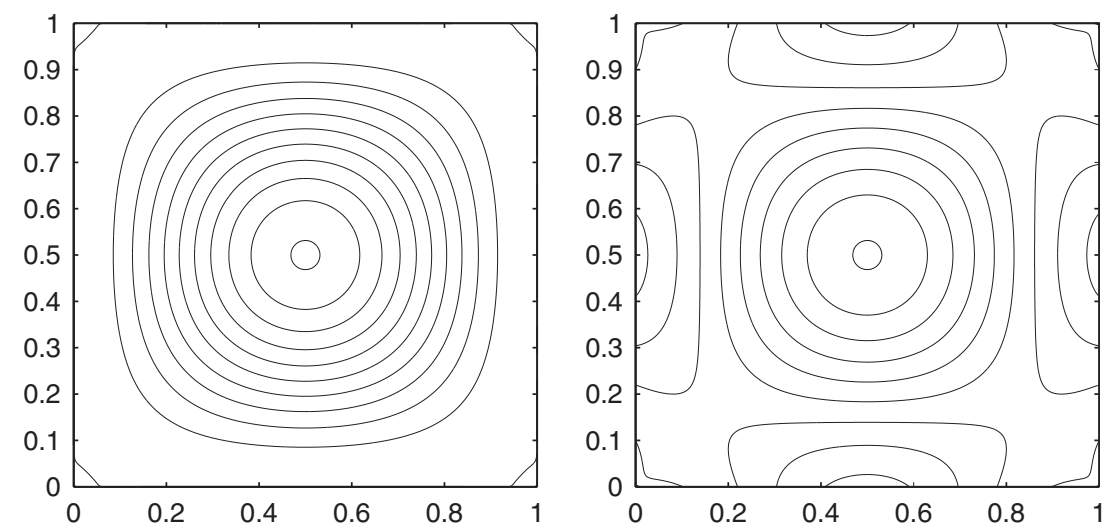

Figure 4. Eigenfunction corresponding to the smallest eigenvalue of the biharmonic eigenvalue problem (left) and buckling plate eigenvalue problem (right).

and the eigenvalue problem for the buckling plate problem is

$$
w=\nabla^{2} \psi, \quad \nabla^{2} \omega=-\lambda \omega, 0<x, y<1, \quad \psi=\frac{\partial \psi}{\partial n}=0 \quad \text { on the boundary }
$$

Both these problems are solved in [23] using a highly accurate spectral Legendre-Galerkin method. The smallest eigenvalue we obtain is compared with that given in [23] in Table I. It can be observed from this table that with an increase in $N$ as well as $M$, the smallest eigenvalue in the present study is found to be approaching the eigenvalue in [23]. With the grid sizes shown in the table agreement up to the fourth decimal place with the values given in [23] are obtained. Further increase in accuracy may be achieved by using a finer grid. The eigenvectors of (14) and (15) corresponding to the eigenvalue on the finest grid size in Table I are shown in Figure 4.

As the eigenvalues of the above-discussed test problems are in good agreement with the literature, the numerical method (as explained in Section 4) is implemented on the LDC and the obtained results are discussed in the next section. 


\section{GLOBAL STABILITY ANALYSIS: RESULTS AND DISCUSSION}

Numerical experiments were carried out on the LDC with aspect ratios $A=1.0,1.5$ and 2.0 to determine the critical Reynolds number, $R e_{\mathrm{c}}$. The algorithm used was

(a) The stationary solution for a given $R e$ is computed from Equation (4).

(b) This stationary solution is substituted in Equations (5) for computation of eigenvalues.

(c) If there is no eigenvalue to the right of the real axis, the $R e$ value is increased and then we return to (a).

(d) If a pair of eigenvalues has a positive real part, then the value of $R e$ is reduced and return to (a).

Steps (a), (b), (c) or (a), (b), (d) are repeated until there exists only one pair of eigenvalues that has zero real part for which the corresponding $R e$ is termed as $R e_{\mathrm{c}}$ and the eigenvalues as critical eigenvalues $\lambda_{\mathrm{c}}$.

Let $\Lambda_{j-1}$ be the largest real part of the eigenvalue whose sign is negative at $R e_{j-1}$ and $\Lambda_{j}$ be the largest real part of the eigenvalue whose sign is positive at $R e_{j}$. Then the following relation [24] was used to choose the next value of $R e$ :

$$
R e=R e_{j-1}+\frac{\left(R e_{j}-R e_{j-1}\right) \Lambda_{j-1}}{\Lambda_{j-1}-\Lambda_{j}}
$$

The steps in the above-mentioned algorithm were then followed to determine if this is the critical Reynolds number at which the Hopf bifurcation occurs.

This algorithm was implemented on various grid sizes with $61 \leqslant N \leqslant 201$ and $251 \leqslant M \leqslant 501$ in order to check the convergence of $R e_{\mathrm{c}}$. The computations were carried out until the convergence was obtained in the critical values.

Contours of the streamlines and vorticity, taken from the finest grid, for the base flow are shown in Figures 6, 7 and 16. Although the stream function contours are smooth, the vorticity contours show minute wiggles (for example, the -2 . level for $A=1.5$ ), which can be shown to diminish if even finer grids are used. With our technique it is not possible to indefinitely keep on increasing
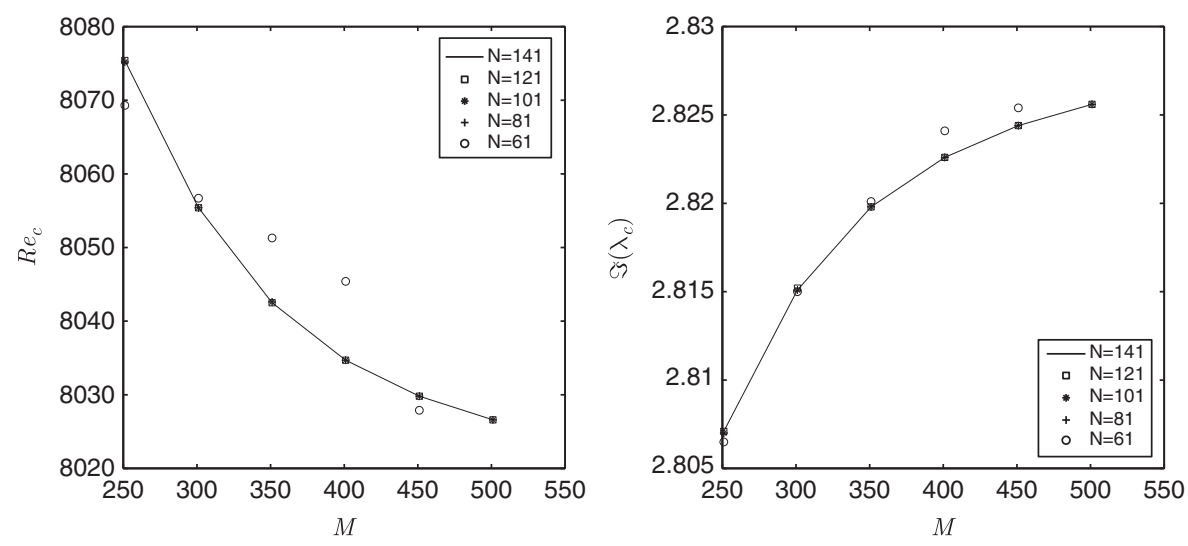

Figure 5. Critical values' dependence on gridsize $(A=1.0)$. 


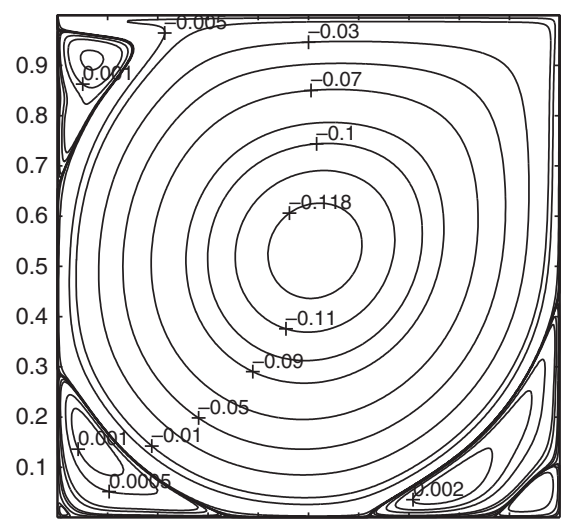

(a)

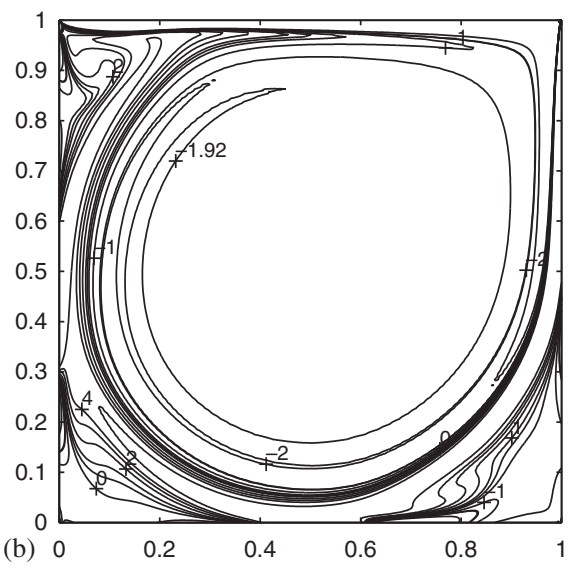

Figure 6. Contours of base flow at $R e_{\mathrm{c}}=8026.6$ for $A=1.0(M=501, N=121)$ : (a) streamlines and (b) equi-vorticity lines.
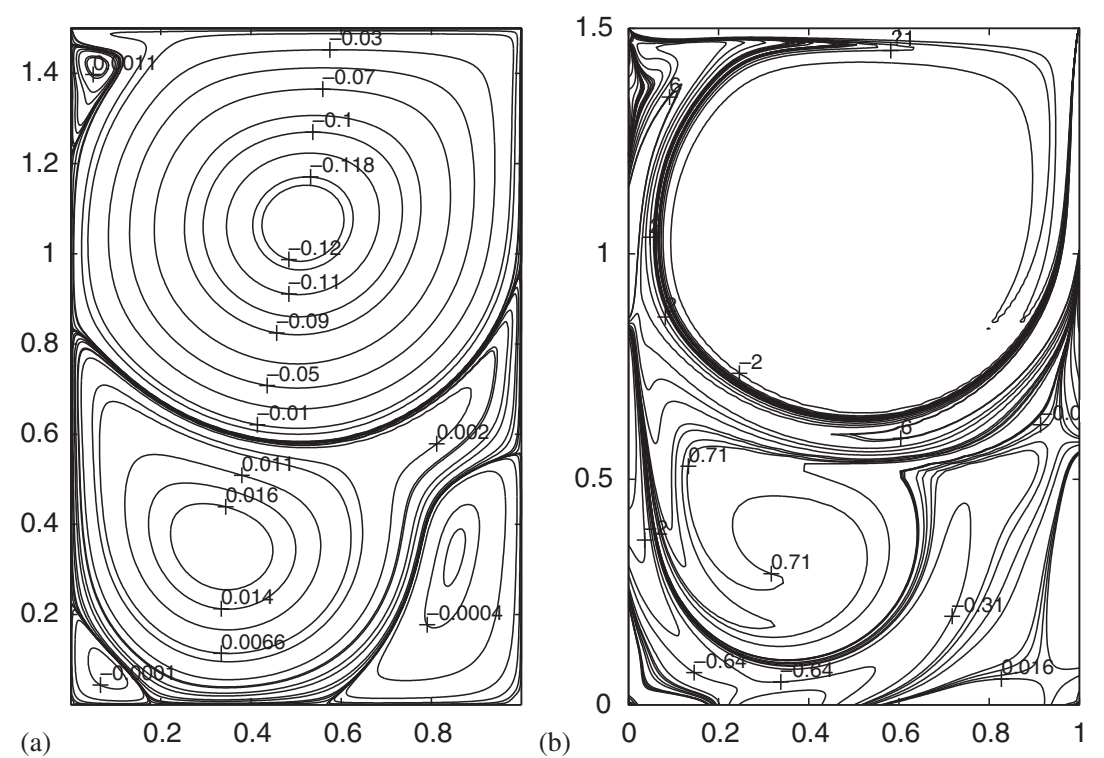

Figure 7. Contours of base flow at $R e_{\mathrm{c}}=5326.9$ for $A=1.5(M=501, N=141)$ : (a) streamlines and (b) equi-vorticity lines.

$N$ because for very large $N$ the Chebyshev differentiation matrices are prone to becoming badly conditioned, and this can generate additional numerical errors. Our results also indicate that the stationary flow first needs to be computed more accurately before being used in the global stability analysis.

The critical values of $\lambda_{\mathrm{c}}$ and $R e_{\mathrm{c}}$ for varying grid sizes are tabulated in Tables II, III and IV and shown in Figures 5, 10 and 15. In Figures 8, 9, 13, 14, 18 and 19 the real and imaginary parts of the stream function and vorticity perturbation eigenvectors at the critical values are shown. These have 
GLOBAL FLOW INSTABILITY IN AN LDC

Table II. Numerical results for $A=1.0$ where the first Hopf bifurcation occurs.

\begin{tabular}{|c|c|c|c|c|c|c|c|c|}
\hline \multirow[b]{2}{*}{$M$} & \multicolumn{2}{|c|}{$N=61$} & \multicolumn{2}{|c|}{$N=81$} & \multicolumn{2}{|c|}{$N=101$} & \multicolumn{2}{|c|}{$N=121$} \\
\hline & $R e_{\mathrm{c}}$ & $\lambda_{c}$ & $R e_{\mathrm{c}}$ & $\lambda_{c}$ & $R e_{\mathrm{c}}$ & $\lambda_{\mathrm{c}}$ & $R e_{\mathrm{c}}$ & $\lambda_{\mathrm{c}}$ \\
\hline 251 & 8069.3 & \pm 2.8065 & 8075.2 & $\pm 2.807 \mathrm{i}$ & 8075.4 & $\pm 2.8071 \mathrm{i}$ & 8075.4 & $\pm 2.8071 \mathrm{i}$ \\
\hline 301 & 8056.7 & \pm 2.8150 & 8055.4 & $\pm 2.8151 \mathrm{i}$ & 8055.4 & $\pm 2.8152 \mathrm{i}$ & 8055.4 & $\pm 2.8151 \mathrm{i}$ \\
\hline 351 & 8051.3 & \pm 2.8201 & 8042.6 & $\pm 2.8198 \mathrm{i}$ & 8042.5 & $\pm 2.8198 \mathrm{i}$ & 8042.5 & $\pm 2.8198 \mathrm{i}$ \\
\hline 401 & 8045.4 & \pm 2.8241 & 8034.7 & $\pm 2.8226 \mathrm{i}$ & 8034.7 & $\pm 2.8226 \mathrm{i}$ & 8034.7 & $\pm 2.8226 \mathrm{i}$ \\
\hline 451 & 8027.9 & \pm 2.8254 & 8029.8 & $\pm 2.8244 \mathrm{i}$ & 8029.8 & $\pm 2.8244 \mathrm{i}$ & 8029.8 & $\pm 2.8244 \mathrm{i}$ \\
\hline 501 & - & - & 8026.6 & $\pm 2.8256 \mathrm{i}$ & 8026.6 & $\pm 2.8256 \mathrm{i}$ & 8026.6 & $\pm 2.8256 \mathrm{i}$ \\
\hline
\end{tabular}

Table III. Numerical results of $A=1.5$ where the first Hopf bifurcation occurs.

\begin{tabular}{|c|c|c|c|c|c|c|}
\hline \multirow[b]{2}{*}{$M$} & \multicolumn{2}{|c|}{$N=101$} & \multicolumn{2}{|c|}{$N=121$} & \multicolumn{2}{|c|}{$N=141$} \\
\hline & $R e_{\mathrm{c}}$ & $\lambda_{\mathrm{c}}$ & $R e_{\mathrm{c}}$ & $\lambda_{\mathrm{c}}$ & $R e_{\mathrm{c}}$ & $\lambda_{\mathrm{c}}$ \\
\hline 251 & 5296.6 & $\pm 2.1352 \mathrm{i}$ & 5293.3 & $\pm 2.1352 \mathrm{i}$ & 5293.2 & $\pm 2.1352 \mathrm{i}$ \\
\hline 301 & 5313.4 & $\pm 2.1365 \mathrm{i}$ & 5309.5 & $\pm 2.1365 \mathrm{i}$ & 5309.5 & $\pm 2.1365 \mathrm{i}$ \\
\hline 351 & 5321.9 & $\pm 2.1372 \mathrm{i}$ & 5317.6 & $\pm 2.1372 \mathrm{i}$ & 5317.6 & $\pm 2.1372 \mathrm{i}$ \\
\hline 401 & 5326.7 & $\pm 2.1376 \mathrm{i}$ & 5322.2 & $\pm 2.1376 \mathrm{i}$ & 5322.3 & $\pm 2.1377 \mathrm{i}$ \\
\hline 451 & 5329.6 & $\pm 2.1379 \mathrm{i}$ & 5325.0 & $\pm 2.1379 \mathrm{i}$ & 5325.1 & $\pm 2.1379 \mathrm{i}$ \\
\hline 501 & 5331.4 & $\pm 2.1380 \mathrm{i}$ & 5326.8 & $\pm 2.1380 \mathrm{i}$ & 5326.9 & $\pm 2.1381 \mathrm{i}$ \\
\hline
\end{tabular}

Table IV. Numerical results of $A=2.0$ where the first Hopf bifurcation occurs.

\begin{tabular}{|c|c|c|c|c|c|c|c|c|}
\hline \multirow[b]{2}{*}{$M$} & \multicolumn{2}{|c|}{$N=141$} & \multicolumn{2}{|c|}{$N=161$} & \multicolumn{2}{|c|}{$N=181$} & \multicolumn{2}{|c|}{$N=201$} \\
\hline & $R e_{\mathrm{c}}$ & $\lambda_{\mathrm{c}}$ & $R e_{\mathrm{c}}$ & $\lambda_{\mathrm{c}}$ & $R e_{\mathrm{c}}$ & $\lambda_{\mathrm{c}}$ & $R e_{\mathrm{c}}$ & $\lambda_{\mathrm{c}}$ \\
\hline 251 & 5904 & $\pm 3.80299 \mathrm{i}$ & 5901 & $\pm 3.80252 \mathrm{i}$ & 5901 & $\pm 3.80263 \mathrm{i}$ & 5901 & $\pm 3.80261 \mathrm{i}$ \\
\hline 301 & 5886 & $\pm 3.80973 \mathrm{i}$ & 5882 & $\pm 3.80927 \mathrm{i}$ & 5882 & $\pm 3.80937 \mathrm{i}$ & 5882 & $\pm 3.80935 \mathrm{i}$ \\
\hline 351 & 5877 & $\pm 3.81332 \mathrm{i}$ & 5872 & $\pm 3.81285 \mathrm{i}$ & 5873 & $\pm 3.81299 \mathrm{i}$ & 5873 & $\pm 3.81298 \mathrm{i}$ \\
\hline 401 & 5871 & $\pm 3.81533 \mathrm{i}$ & 5867 & $\pm 3.81493 \mathrm{i}$ & 5867 & $\pm 3.81502 \mathrm{i}$ & 5867 & $\pm 3.81501 \mathrm{i}$ \\
\hline 451 & 5868 & $\pm 3.81658 \mathrm{i}$ & 5863 & $\pm 3.81614 \mathrm{i}$ & 5864 & $\pm 3.81628 \mathrm{i}$ & 5864 & $\pm 3.81267 \mathrm{i}$ \\
\hline 501 & 5866 & $\pm 3.81738 \mathrm{i}$ & 5861 & $\pm 3.81695 \mathrm{i}$ & 5861. & $\pm 3.81704 \mathrm{i}$ & 5861 & $\pm 3.81702 \mathrm{i}$ \\
\hline
\end{tabular}

been normalized so that the maximum absolute value of the function is unity. Though the steady flow contours are relatively smooth, the eigenvector plots do show more wiggles, particularly for the larger aspect ratios. These again can be shown to decrease with finer grids.

In Figure 8, an alternate band of positive and negative streamlines can be observed in the perturbation eigenvectors all along the left boundary. A similar behavior is also observed clearly in the case of real and imaginary parts of vorticity eigenvector in Figure 9. This kind of pattern was observed by Auteri et al. [15] for aspect ratio $A=1$ in the time sequence of fluctuating vorticity field at $R e=8125$, the value above the critical Reynolds number. They stated that this could be due to shear layer instability due to which the primary vortex is entirely separated from the secondary vortex and the cavity walls. 

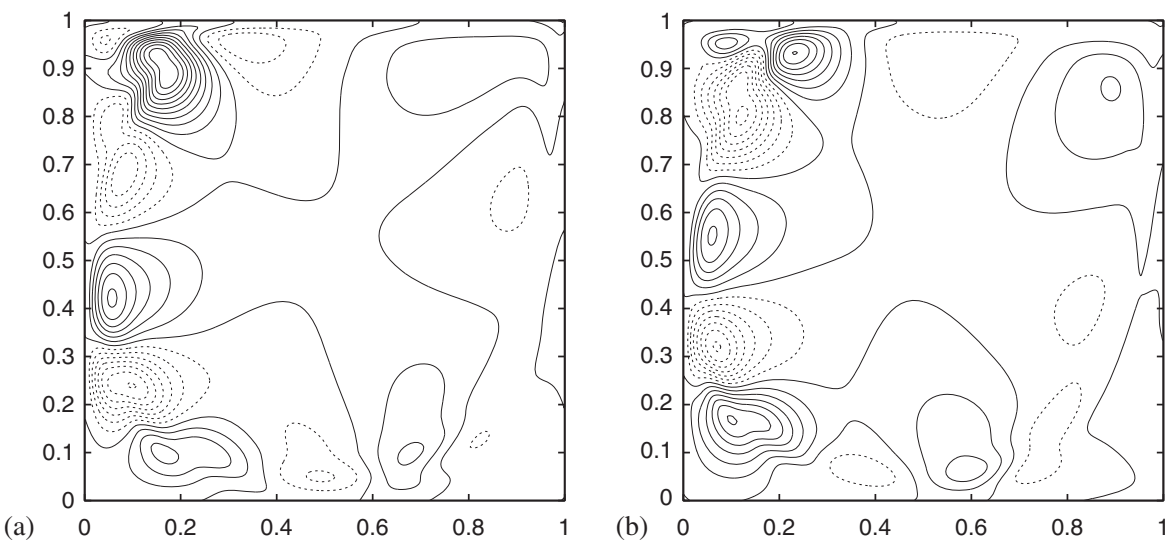

Figure 8. Contours of the (a) real and (b) imaginary parts of the stream function eigenvector at $R e_{\mathrm{c}}=8026.6$ for $A=1.0(M=501, N=121)$. Contour levels shown are in increments of 0.1 with negative contour values shown by the dashed lines.
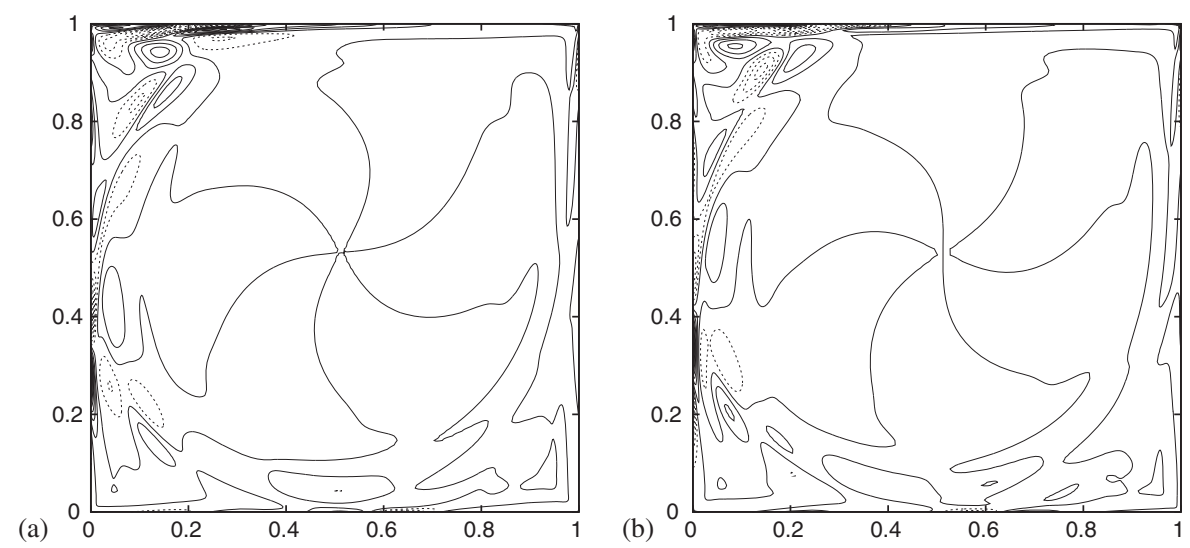

Figure 9. Contours of the (a) real and (b) imaginary parts of the vorticity function eigenvector at $R e_{\mathrm{c}}=8026.6$ for $A=1.0(M=501, N=121)$. Contour levels shown are in increments of 0.1 with negative contour values shown by the dashed lines.

Some additional points that may be inferred from the data are given below separately for each aspect ratio.

Case $1(A=1.0)$. It is noticed from Figure 5 that while $R e_{\mathrm{c}}$ decreases with increase in $M$ for a fixed $N, \Im\left(\lambda_{\mathrm{c}}\right)$ is found to be increasing very slowly. For each of the grid values given in Table II, the number of eigenvalues calculated is 100 using 1000 basis vectors and a shift value of $\sigma=3.0$. It was found that the $R e_{\mathrm{c}}$ values for $N=61, M=501$ were affected by the presence of a spurious eigenvalue (giving $R e_{\mathrm{c}}=7849$ ) and for this reason it is not tabulated.

Figure 11(a) shows the eigenvalue spectrum at the critical Reynolds number. The eigenvalues crossing the imaginary axis and that cause the flow in the LDC to be neutrally stable are clearly 


\section{GLOBAL FLOW INSTABILITY IN AN LDC}
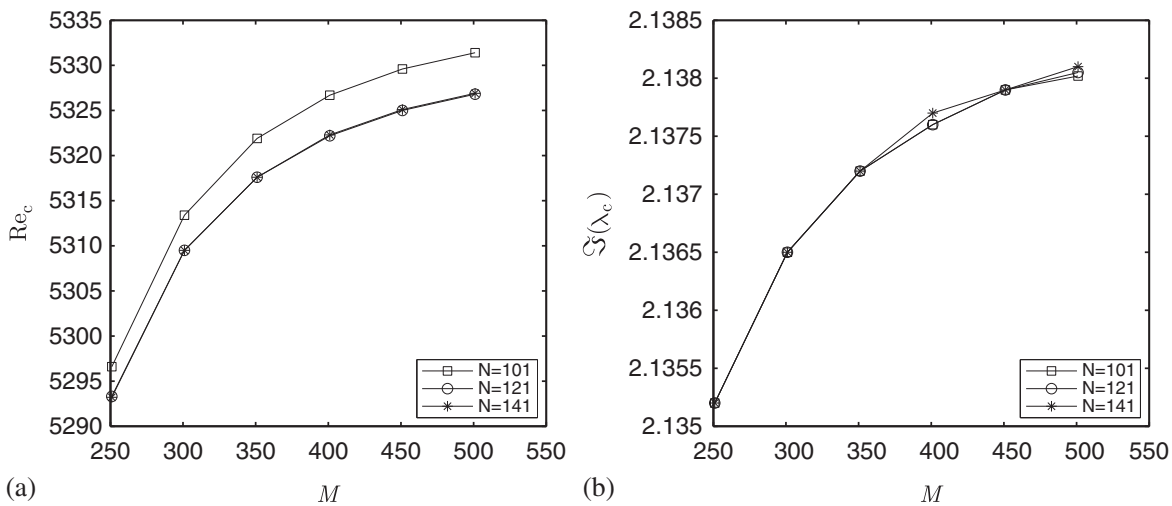

Figure 10. Critical values dependence on grid size $(A=1.5)$.
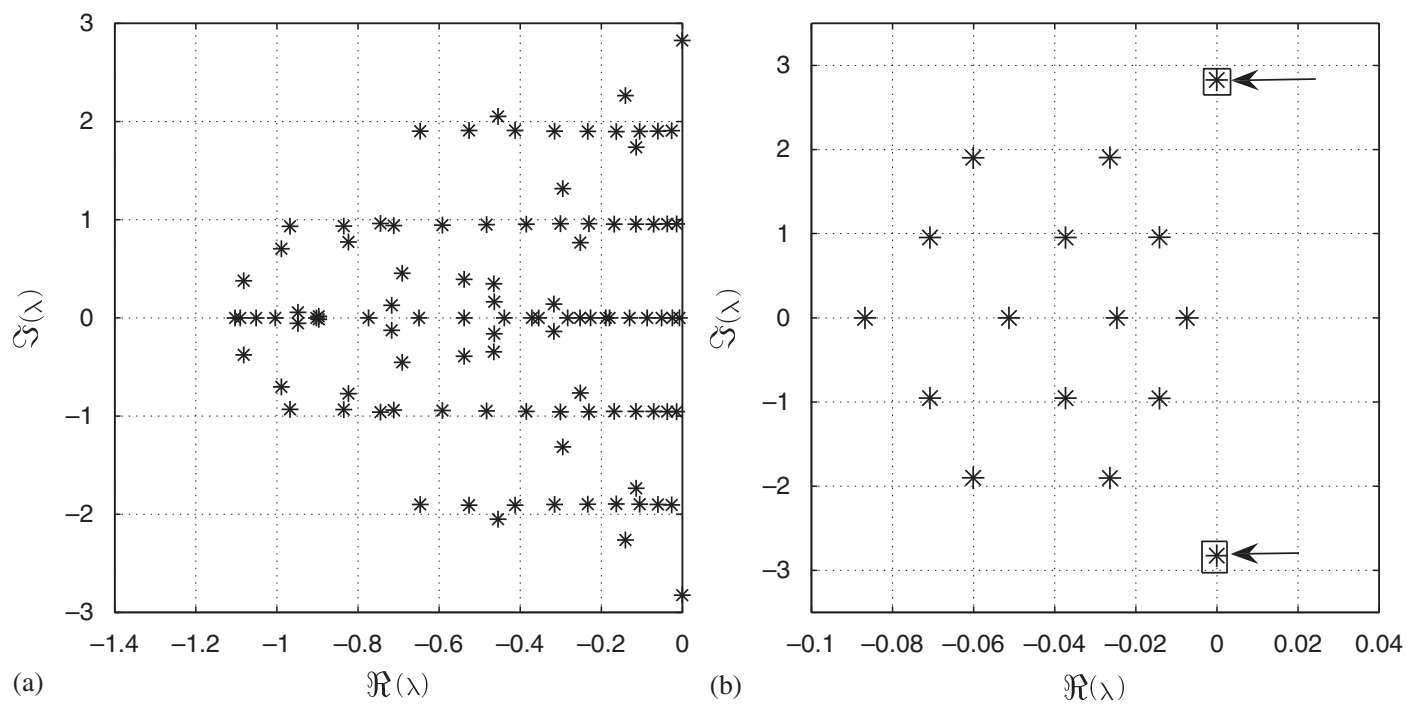

Figure 11. Plots showing (a) 100 eigenvalues and (b) few eigenvalues near the imaginary axis at $R e_{\mathrm{c}}=8026.6$ for $A=1.0(M=501, N=121)$.

shown in Figure 11(b). A cubic spline fit of the obtained numerical data estimated the value of the critical parameter corresponding to zero mesh size $\left(\operatorname{Re}_{\mathrm{c}}(h=0.001)\right)$ as 8020.1 .

Reducing $N$ values further created additional problems in that the base flow could not be computed sufficiently accurately and problems with convergence were experienced for increasing Reynolds numbers. Clearly the eddy boundaries cannot be accurately resolved with a reduced number of grid points and this is one of the reasons for this difficulty.

Case $2(A=1.5)$. The values of $\lambda_{\mathrm{c}}$ and $R e_{\mathrm{c}}$ for different grid sizes tabulated in Table III were obtained by calculating 200 eigenvalues using 1000 basis vectors with $\sigma=3$. It can be inferred 

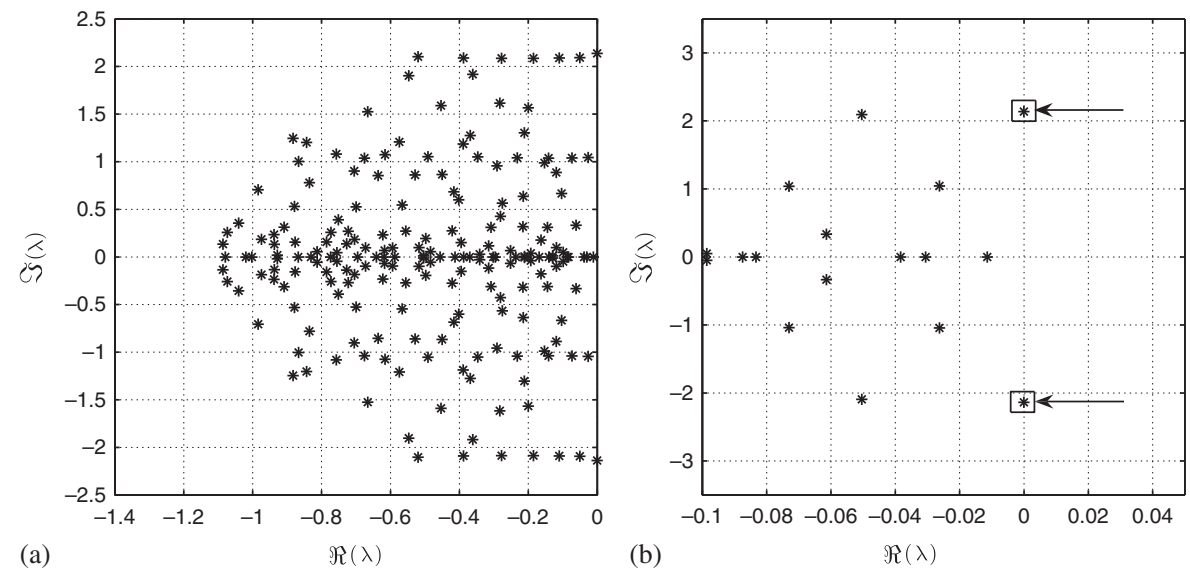

Figure 12. Plots showing (a) 200 eigenvalues and (b) few eigenvalues near the imaginary axis at $R e_{\mathrm{c}}=5326.9$ for $A=1.5(M=501, N=141)$.
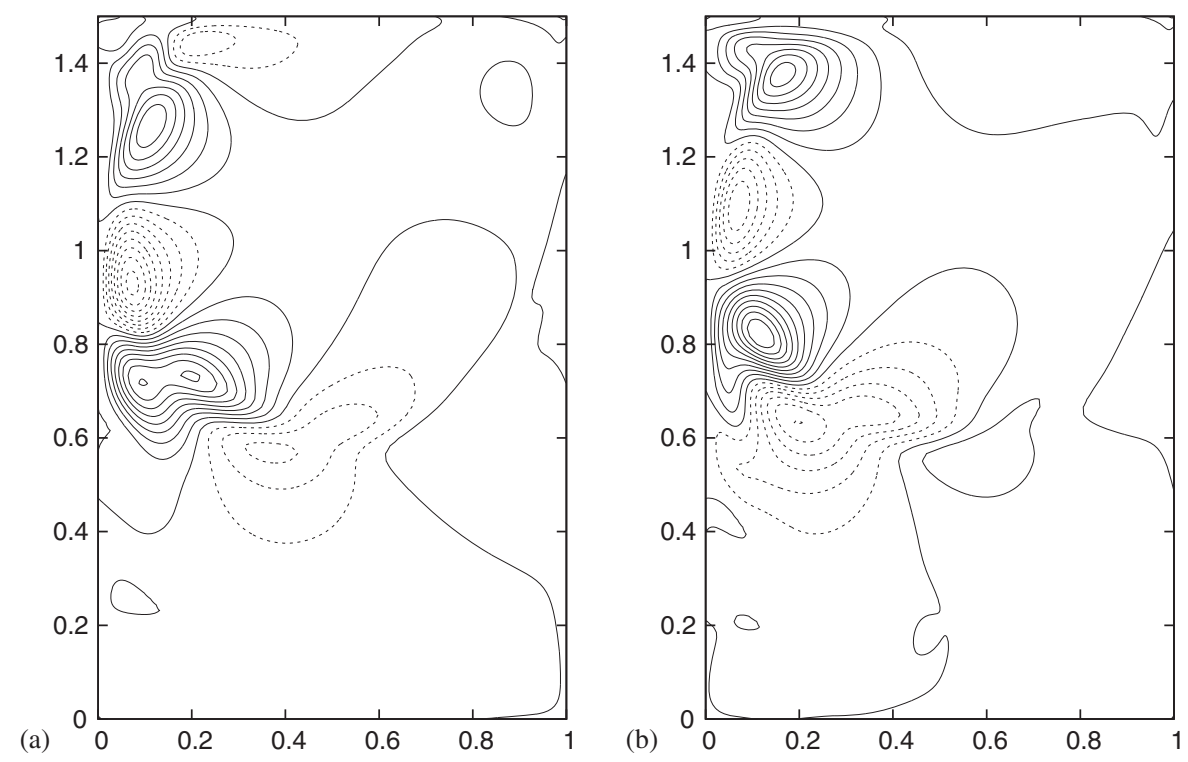

Figure 13. Contours of the (a) real and (b) imaginary parts of the stream function eigenvector at $R e_{\mathrm{c}}=5326.9$ for $A=1.5(M=501, N=141)$. Contour levels shown are in increments of 0.1 with negative contour values shown by the dashed lines.

from this table that for each $M, R e_{\mathrm{c}}$ remained the same for $N=121$ and 141 but differed for $N=101$ unlike $\lambda_{\mathrm{c}}$, which remained the same (except for $M=401$ and 501) for all values of $N$. This is clearly illustrated in Figure 10. Figure 10(a) shows that for a constant $N$, as $M$ increases, there is a gradual increase in $R e_{\mathrm{c}}$ values. An interesting feature is that this behavior of $R e_{\mathrm{c}}$ with increase in $M$ is opposite when compared with that of $A=1.0$. There happened to be a very 


\section{GLOBAL FLOW INSTABILITY IN AN LDC}
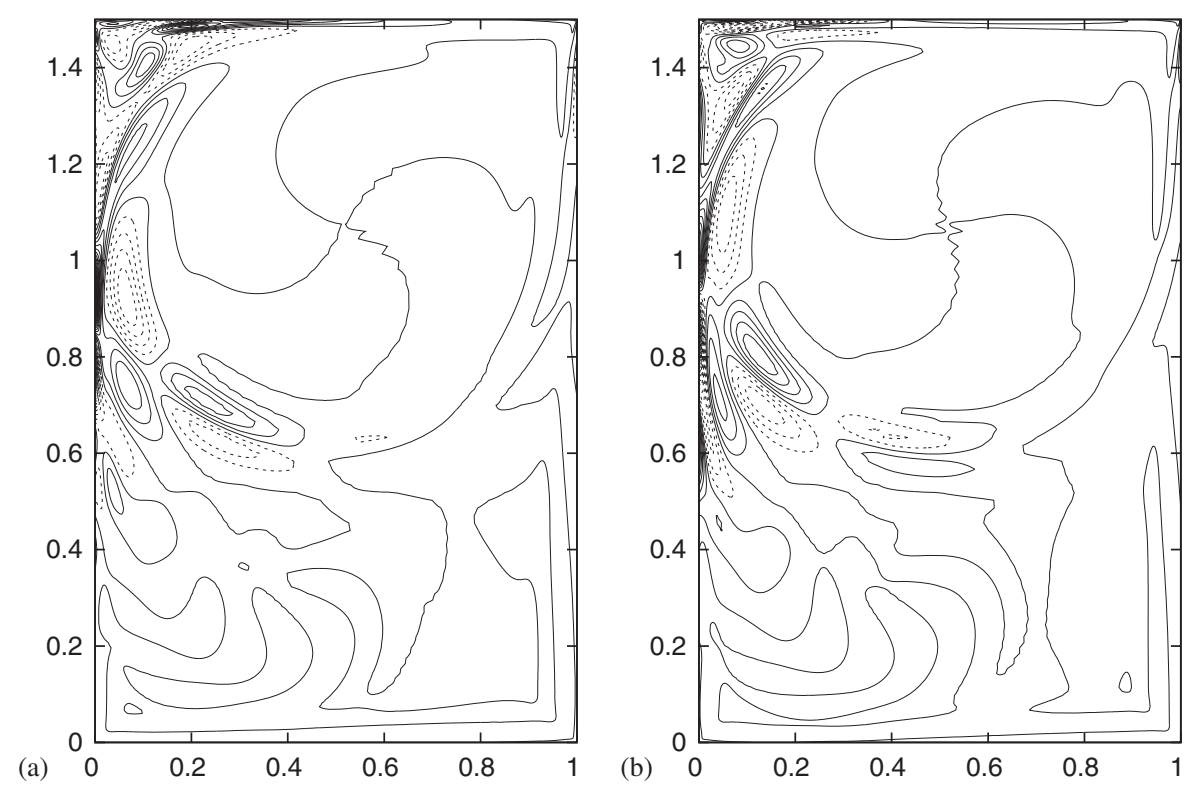

Figure 14. Contours of the (a) real and (b) imaginary parts of the vorticity eigenvector at $R e_{\mathrm{c}}=5326.9$ for $A=1.5(M=501, N=141)$. Contour levels shown are in increments of 0.05 with negative contour values shown by the dashed lines.
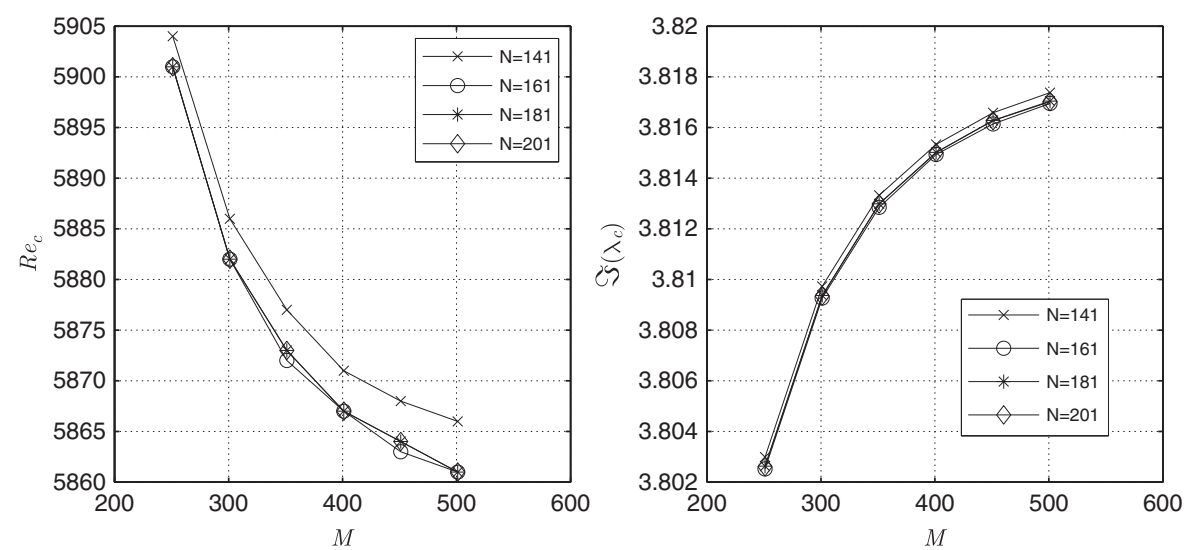

Figure 15. Critical values' dependence on grid size $(A=2.0)$.

slight increase (only in the third decimal place for $M=401$ and $501, N=141$ ) in the values of $\Im\left(\lambda_{\mathrm{c}}\right)$ with increase in $M$ for a constant $N$ as depicted in Figure 10(b). Figure 12(a) illustrates the eigenvalues computed to determine the critical parameter for the finest grid size. The Hopf bifurcation is characterized by a pair of complex conjugate eigenvalues crossing the imaginary axis and is shown clearly in Figure 12(b). The contours of the perturbed flow that are the eigenvectors are shown in Figures 13 and 14. Using the obtained computational results, the critical parameter 

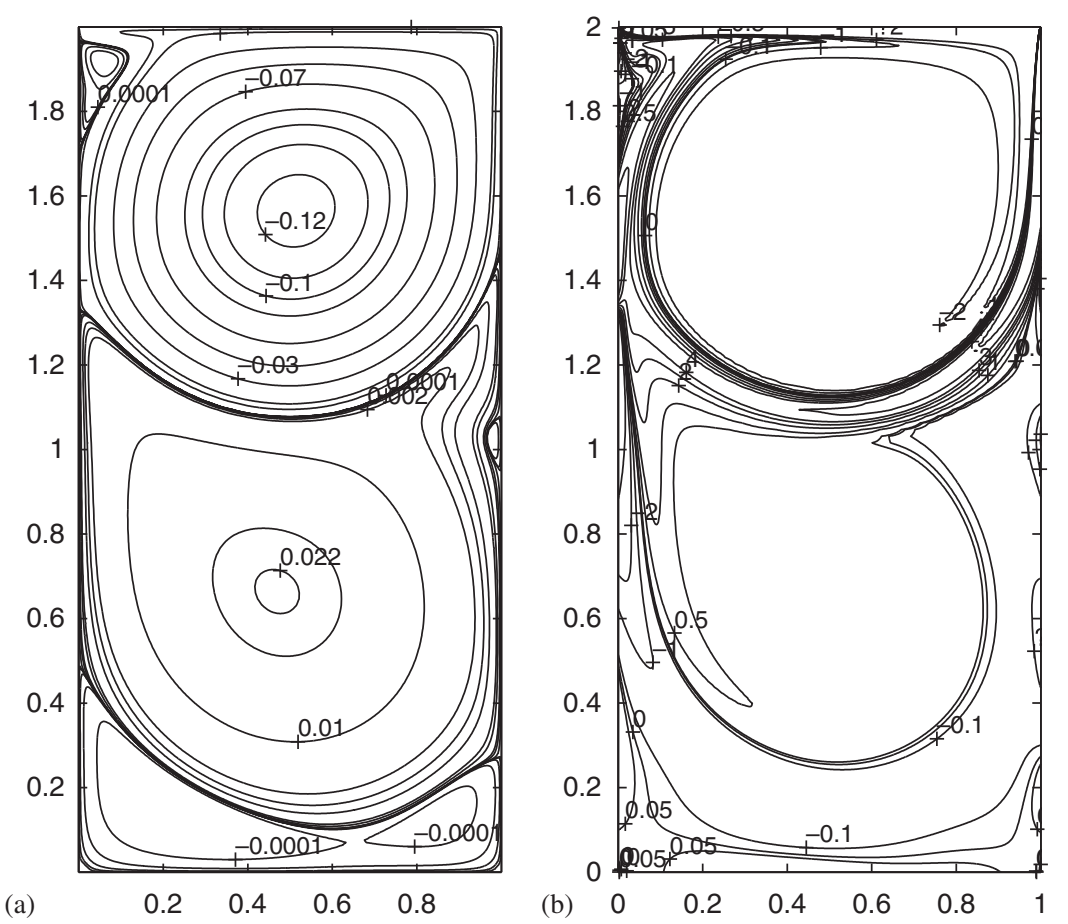

Figure 16. Contours of (a) stream function and (b) vorticity for the base flow at $R e_{\mathrm{c}}=5861$ for $A=2.0(M=501, N=201)$.
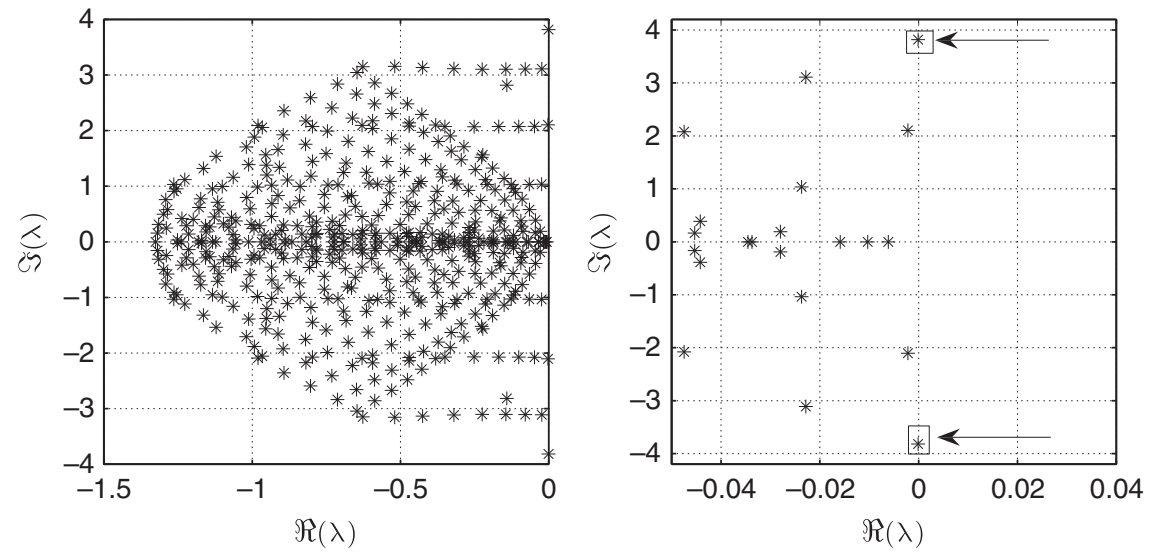

Figure 17. Plots showing the 500 eigenvalues and (right) a closeup of the eigenvalues near the imaginary axis at $R e_{\mathrm{c}}=5861$ for $A=2.0(M=501, N=201)$. 

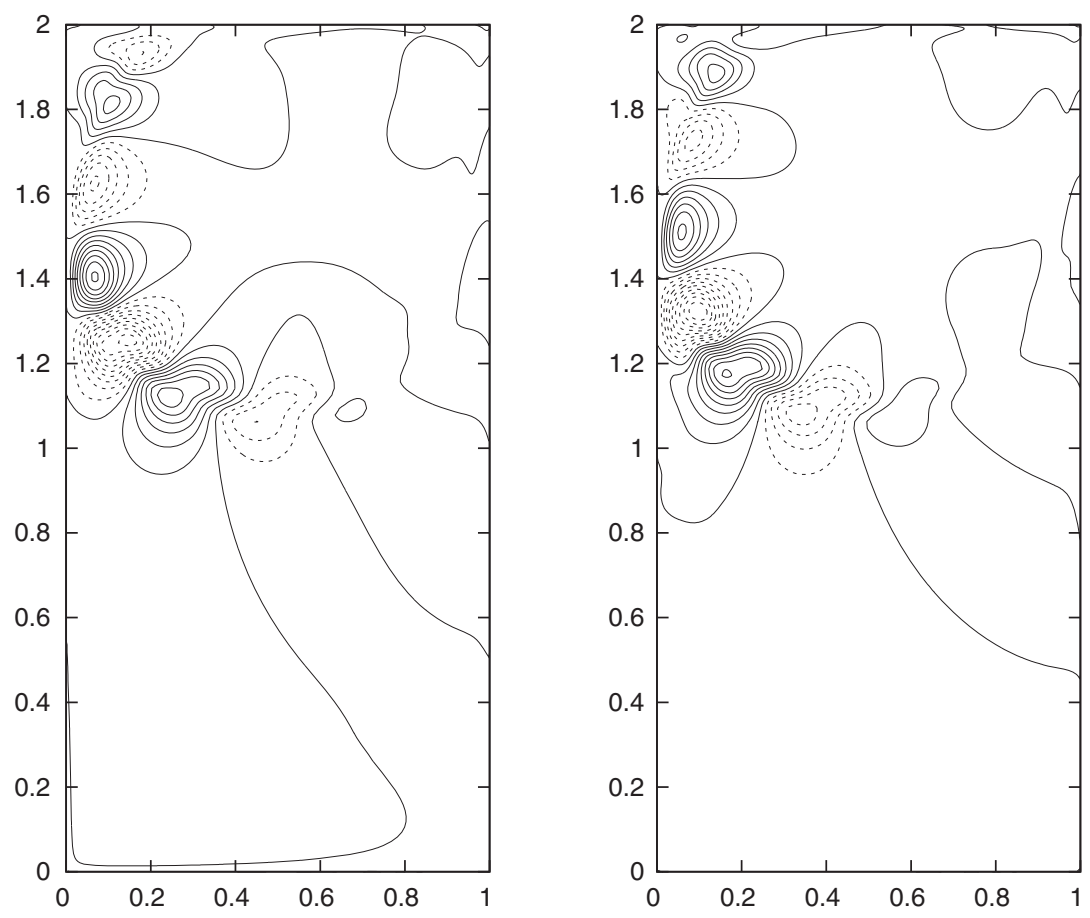

Figure 18. Contours of the real (left) and imaginary parts (right) of the perturbation stream function eigenvector at $R e_{\mathrm{c}}=5861$ for $A=2.0(M=501, N=201)$. Contour levels shown are in increments of 0.1 with negative contour values shown by the dashed lines.

corresponding to the finest mesh size $\operatorname{Re}_{\mathrm{c}}(h=0.001)$ is estimated to be 5331.2 by means of a cubic spline fit.

Case $3(A=2.0)$. The values of $R e_{\mathrm{c}}$ and $\Im\left(\lambda_{\mathrm{c}}\right)$ obtained for different grid sizes are given in Table IV, which were obtained by computing 500 eigenvalues with 1500 basis vectors and $\sigma=6$. It is to be noted here that choosing a smaller value of $\sigma$ does not give the most unstable eigenvalue and instead the results point to a critical value of $\lambda_{c}= \pm 2.103 \mathrm{i}$. On the other hand, our simulations (see later) suggested a different frequency more in agreement with the value calculated by taking larger values of $\sigma$. The dependence of critical values on grid size is depicted in Figure 15. The contours of streamlines and vorticity of the base flow and its perturbed flow at the critical Reynolds number are illustrated in Figures 16, 18 and 19 corresponding to the grid size of $N=201$ and $M=501$. We observe the primary, secondary and tertiary vortices from the top the bottom of the cavity in Figure 16(a). The 500 eigenvalues that were calculated to compute the critical parameter are shown in Figure 17(a). In Figure 17(b), the eigenvalues that correspond to the Hopf bifurcation are clearly shown. Two pairs of alternate rotating vortices are observed on the left side of the top portion of the cavity in Figure 18. The contours of vorticity eigenvector in Figure 19 also clearly show a band of alternate positive and negative values of vorticity along the top left boundary. The wiggles in the central portion of the primary and secondary cavities in these figures indicate the insufficient grid resolution. The cubic spline fitting of the obtained numerical results $(M=251$ to 

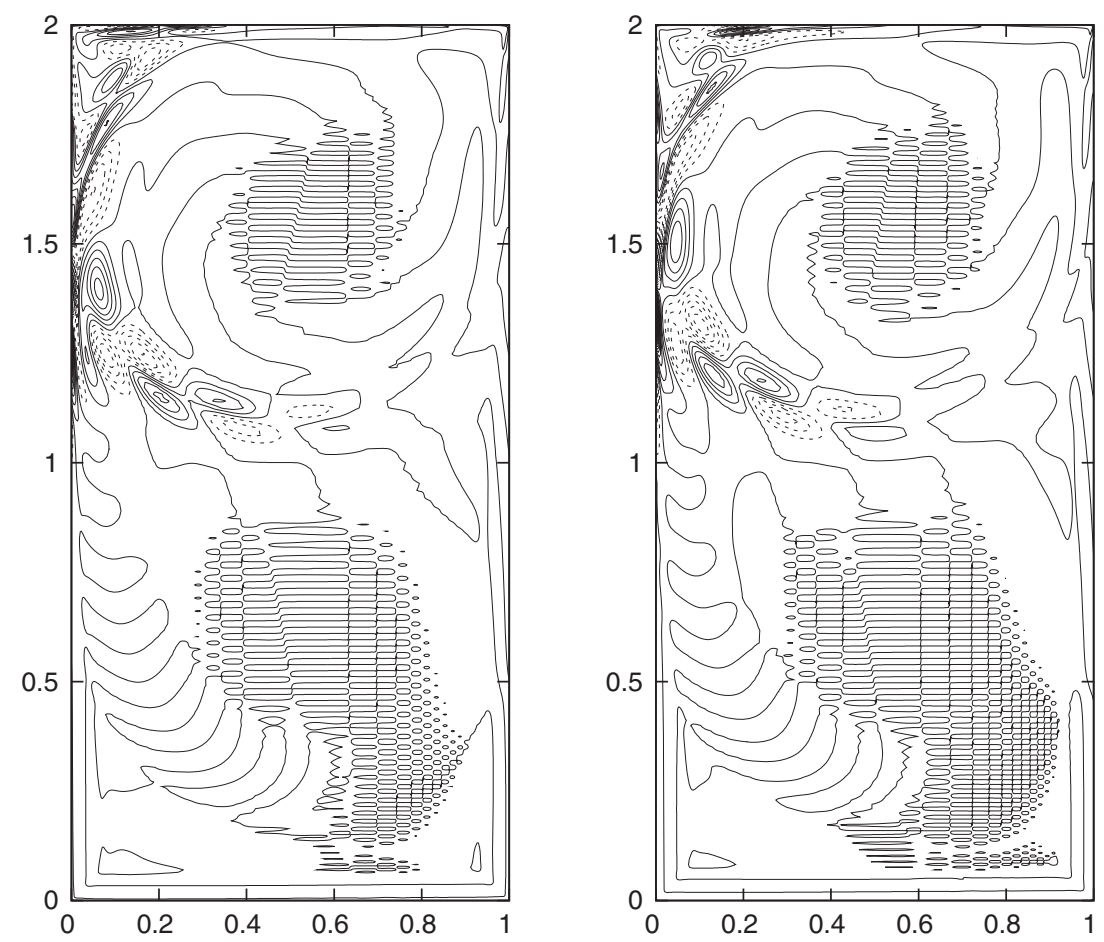

Figure 19. Contours of the real (left) and imaginary parts (right) of the perturbation vorticity eigenvector at $R e_{\mathrm{c}}=5861$ for $A=2.0(M=501, N=201)$. Contour levels shown are in increments of 0.05 with negative contour values shown by the dashed lines.

Table V. Computed values of frequencies $f_{s}$ from simulations and $f_{g}$ from the global stability analysis.

\begin{tabular}{lllcc}
\hline$A$ & $N \times M$ & $R e_{\mathrm{c}}$ & $f_{s}$ & $f_{g}$ \\
\hline 1.0 & $81 \times 501$ & 8026.7 & 0.4480 & 0.4467 \\
1.5 & $141 \times 501$ & 5326.9 & 0.3400 & 0.3398 \\
2.0 & $161 \times 501$ & 5861 & 0.6061 & 0.6075 \\
\hline
\end{tabular}

501 and $N=201)$ estimated the value of $\operatorname{Re}_{\mathrm{c}}(h=0.001)$ as 5790 , whereas linear extrapolation yields 5847.5.

Linear temporal simulations were also performed wherein the frequencies obtained from the global stability analysis $\left(f_{g}=\Im\left(\lambda_{\mathrm{c}}\right) / 2 \pi\right)$ are compared and explained in the following section.

\subsection{Linear temporal simulation: results and discussion}

Although the global stability analysis on various grid sizes for the three configurations exhibited a gradual increasing trend in $\Im\left(\lambda_{c}\right)$, with increase in $M$ and for any given $N$, the difference in the successive values is small, see Figures 5(b), 10(b) and 15(b). Hence, the linear temporal simulation 

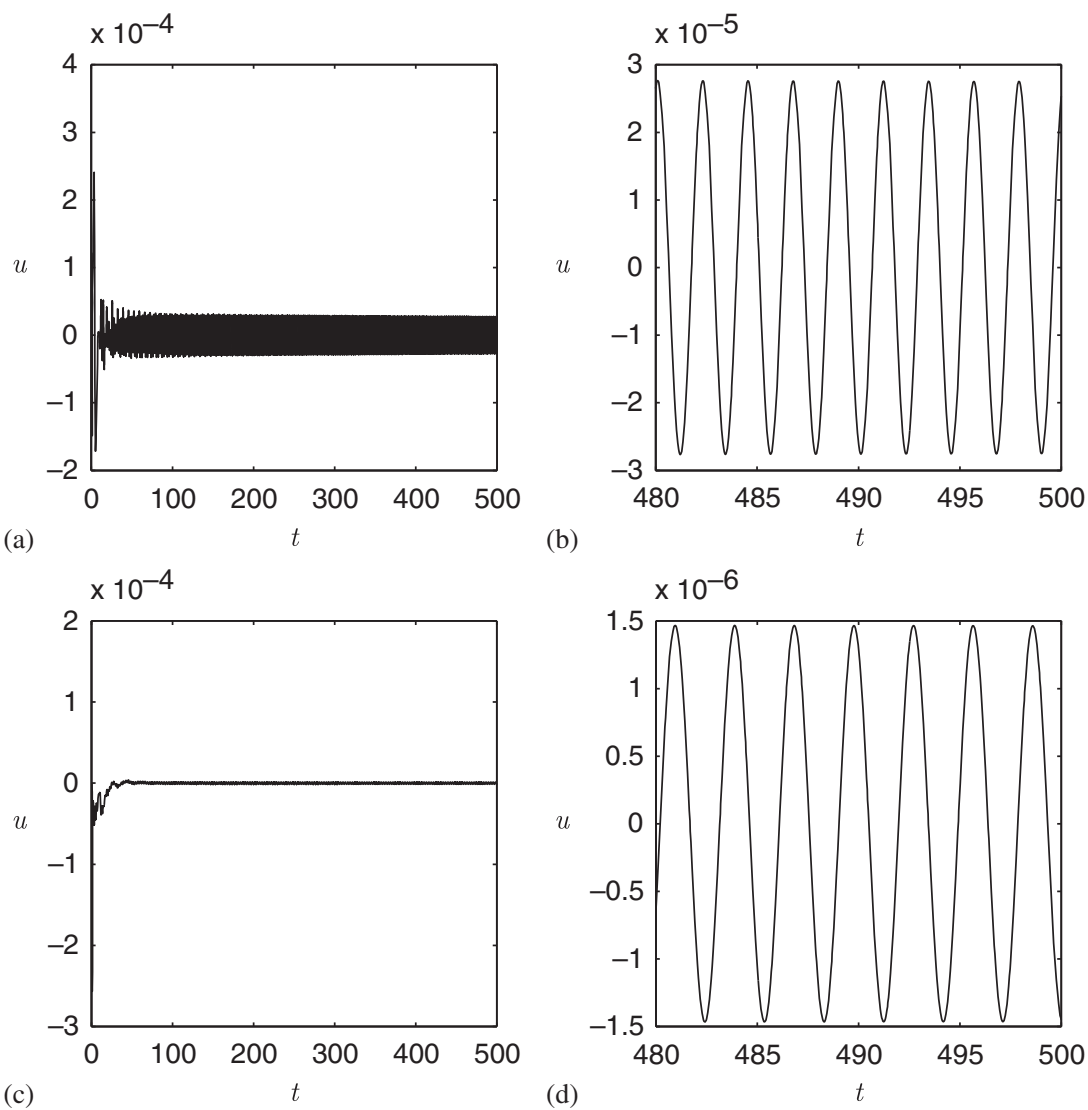

Figure 20. Evolution of $u$ with $t$ (left) and a snapshot of the same signal (right) for $A=1.0, M=501$, $N=81, \operatorname{Re}_{\mathrm{c}}=8026.7((\mathrm{a}),(\mathrm{b}))$ and $A=1.5, M=501, N=141, \operatorname{Re}_{\mathrm{c}}=5326.9$ ((c),(d)).

was performed on one grid size (as given in the figure captions) from each of the configurations. Before proceeding with the simulation, a numerical stability check was also performed to ensure that our simulation results were completely devoid of numerical instabilities. For the simulations the linearized unsteady Navier-Stokes equations (5) were solved. A disturbance of Gaussian form in space and time with

$$
\tilde{\psi}(x, y=0, t)=C \mathrm{e}^{-50(t-1)^{2}} \mathrm{e}^{-20(x-(1 / 2))^{2}}, \quad t>0
$$

was imposed and allowed to evolve. After an initial time step, a second-order in-time fully implicit scheme was used and the same spatial discretizations as described earlier for the global analysis were used. In order to choose a proper time step to compute the frequency of the flow in the LDC, at first simulations were carried out up to time $t=100$ by using different time steps on a grid size of $81 \times 251$ at $R e=8000$ for $A=1.0$. Goodrich et al. [14] have mentioned that the velocity component at a point serves as one of the dynamic measures to track the convergence to an asymptotic state and to understand the qualitative nature of that state. For this reason, the horizontal 

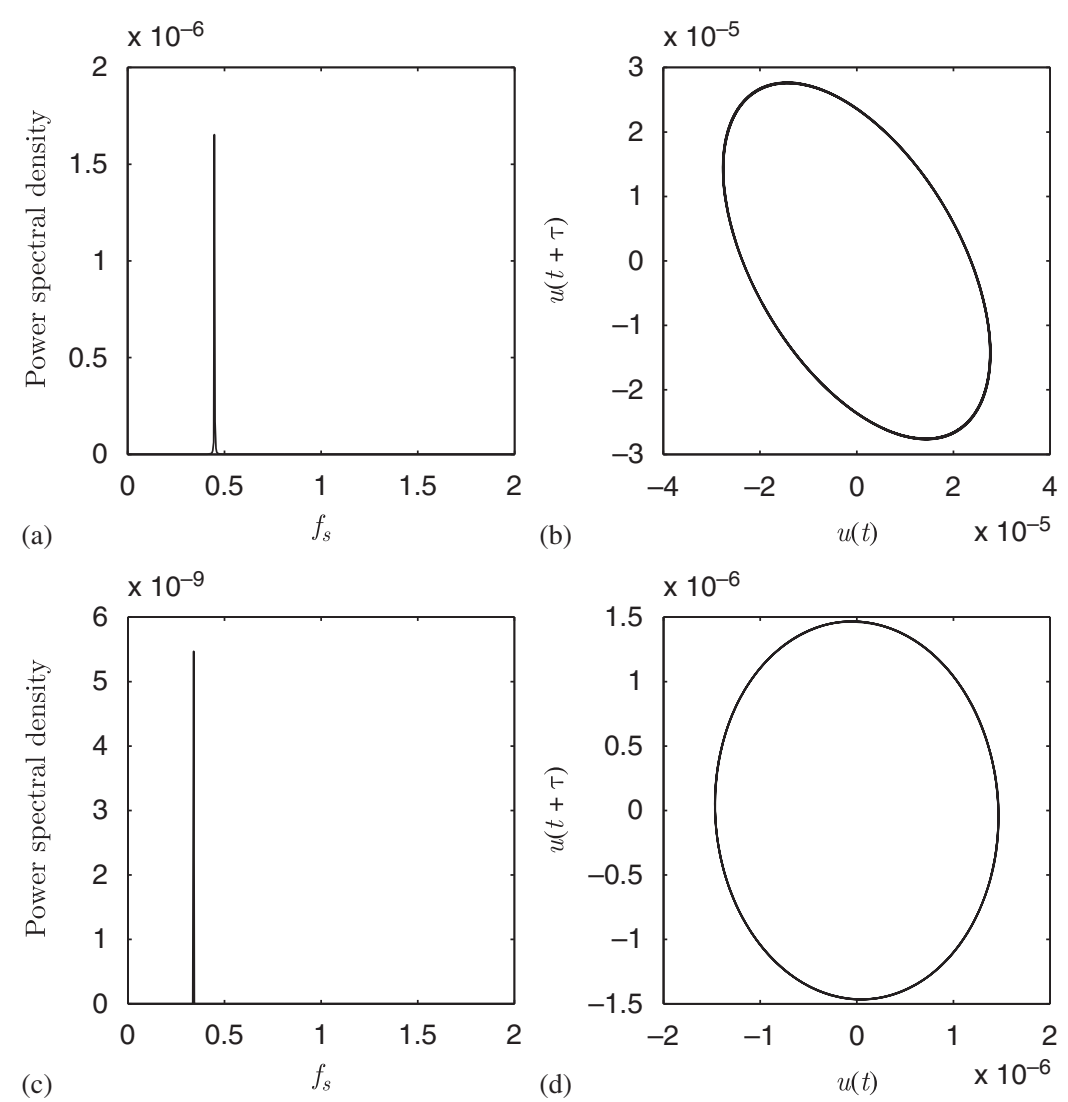

Figure 21. Power spectral density (left) and phase portrait (right) of $u$ with $\tau=0.75$ for $A=1.0, M=501$, $N=81, R e_{\mathrm{c}}=8026.7((\mathrm{a}),(\mathrm{b}))$ and $A=1.5, M=501, N=141, R e_{\mathrm{c}}=5326.9$ ((c),(d)).

velocity denoted by ' $u$ ' at an arbitrary node is chosen as a dynamic indicator. By computing the values of $u$ at each time interval for different time steps, it was found that $\Delta t=0.025$ was sufficient to capture the fundamental frequency of the flow.

As mentioned in Section 3, at the Hopf bifurcation, a one parameter family of periodic solution bifurcates from the stationary solution. This implies that the solution has some time-periodic pattern with a certain frequency. The linear temporal simulation was carried out to determine the fundamental frequency $\left(f_{s}\right)$, shown in Table $\mathrm{V}$, which should ideally be close to that obtained from the global stability analysis $\left(f_{g}=\Im\left(\lambda_{\mathrm{c}}\right) / 2 \pi\right)$. Therefore the simulations were performed up to $t=500$ units for $A=1.0$ and 1.5. The evolution of $u$ with time $t$ at the respective values of $R e_{\mathrm{c}}$ is shown in Figure 20(a) and (c). Fourier analysis of the obtained data was done to compute the fundamental frequency. This is shown in the graphs of power spectral density versus frequency in Figure 21. The closed form of the phase portraits in these figures further confirms the periodic nature of the solution. For $A=2$ it was found that there was a large transient response, see Figure 22 and simulations needed to be conducted for a longer time, $t=1500$ units. Again the power spectral density plots in Figure 23 not only confirm the dominant global frequency predicted by the stability 

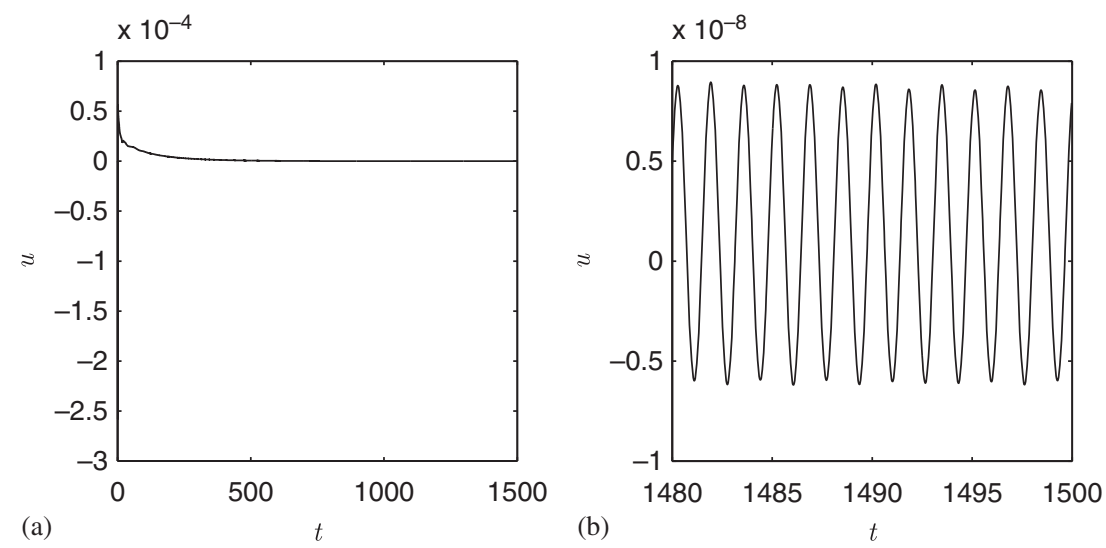

Figure 22. Evolution of $u$ with $t$ (a) and a snapshot of the same signal and (b) for $A=2.0, M=501, N=161, R e_{\mathrm{c}}=5861$.
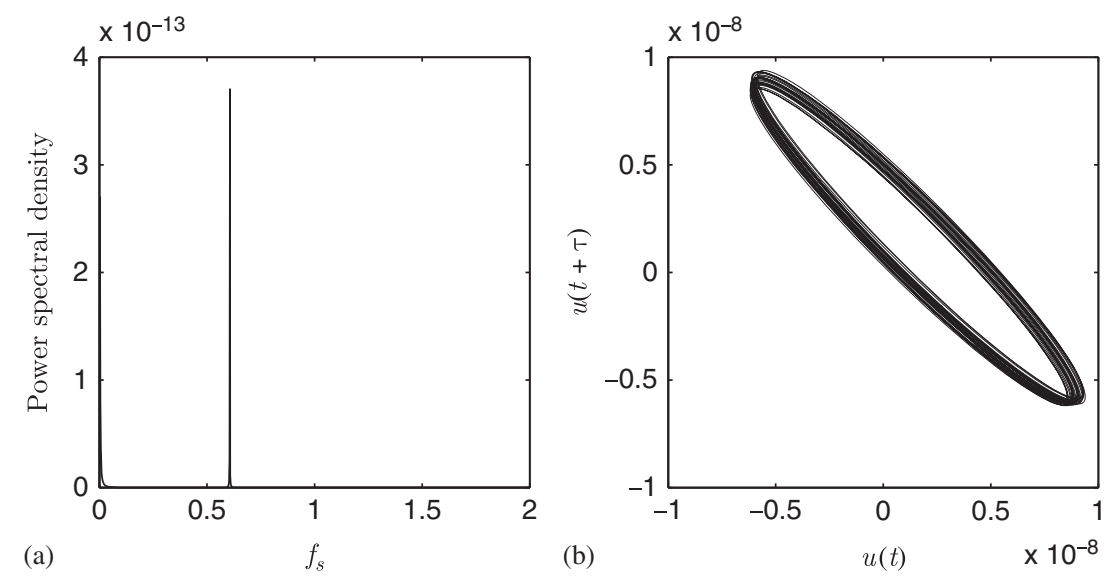

Figure 23. Power spectral density (a) and phase portrait (b) of $u$ with $\tau=0.75$ for $A=2.0, M=501, N=161, R e_{\mathrm{c}}=5861$.

analysis but the power spectrum also shows significant peak for low frequencies emanating from the transient response.

The stream function perturbation at the Hopf point may be expressed as

$$
\tilde{\psi}=A\left(\tilde{\psi}_{r} \cos \left(\lambda_{i} t+\phi\right)-\tilde{\psi}_{i} \sin \left(\lambda_{i} t+\phi\right)\right)
$$

where $\tilde{\psi}_{r}, \tilde{\psi}_{i}$ are the real and imaginary parts of the eigenvector, and $A, \phi$ are arbitrary amplitude and phase constants, respectively. By fixing the phase and amplitude at one instant in time to match with the simulation data, the results from the simulations for the rest of the cycle are compared with the predictions from the global analysis in Figures 24, 25 and 26 for the different aspect 

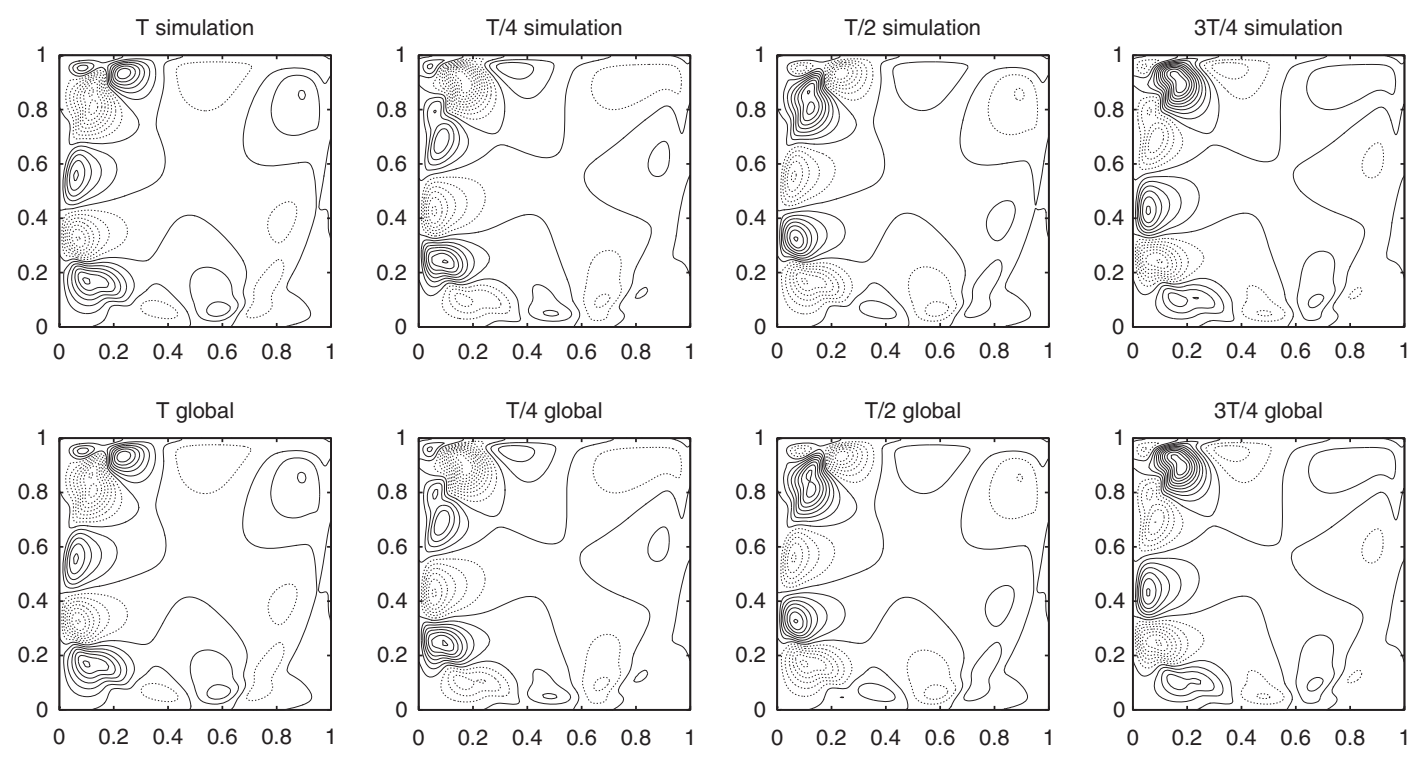

Figure 24. Comparisons of simulations (top row) and global analysis predictions (bottom row) for one period $T$ in intervals of $T / 4$ from left to right for aspect ratio $A=1$ and $R e=8026.7$ with $M=501, N=80$.

Contour levels are in intervals of 0.1 , with negative levels shown by dashed lines.

ratios. The predicted comparisons agree very well with the simulations for $A=1$ and $A=1.5$ and the influence of the transients may be the reason for the slight differences for $A=2$ (Figure 26).

The computational results of $A=1.0,1.5$ and 2.0 are compared with the available literature and are discussed in the next section.

\subsection{Comparison of results with the literature}

Table VI shows the values of $R e_{\mathrm{c}}$ and $\lambda_{\mathrm{c}}$ obtained by various researchers, the methods they adopted and also the grid/mesh sizes they have used.

For the case of $A=1.0$, it can be inferred that the value of $R e_{\mathrm{c}}$ varies between 7704 and 8050 and the value of $\Im\left(\lambda_{\mathrm{c}}\right)$ varies from 2.76 to 2.86 (the accuracy of $\Im\left(\lambda_{\mathrm{c}}\right)=3.85$ obtained by Cazemier $e t$ al. was questioned even by them as it significantly differed from their direct numerical simulation (DNS) approach). Our critical parameter $R e_{\mathrm{c}}=8026.6$ and the associated value of $\Im\left(\lambda_{\mathrm{c}}\right)=2.826$ corresponding to finest grid size $121 \times 501$ lie very much within the range mentioned above. In terms of \% of relative difference, the $R e_{\mathrm{c}}$ value differs by $0.29 \%$ with Bruneau and Saad [27] and with Peng et al. [18], it differs by $4.19 \%$. On the other hand $\Im\left(\lambda_{\mathrm{c}}\right)$ differs by $1.18 \%$ with Poliashenko and Aidun [16] and 2.39\% with Tiesinga et al. [26]. It is observed in Figure 5(b) that there is a very slight change (only in the second and third decimal) in $\Im\left(\lambda_{\mathrm{c}}\right)$ with increase in $M$. A similar independence of critical eigenvalue with grid size was even observed by Fortin et al. [17].

A striking contrast in the values of $R e_{\mathrm{c}}$ corresponding to $A=1.5$ can be observed in Table VI. This table also shows the limited amount of work done for the $A=1.5$ case. The relative difference of $R e_{\mathrm{c}}$ in terms of $\%$ is $26 \%$ with Poliashenko and Aidun [16] and 6.11\% with Abouhamza 

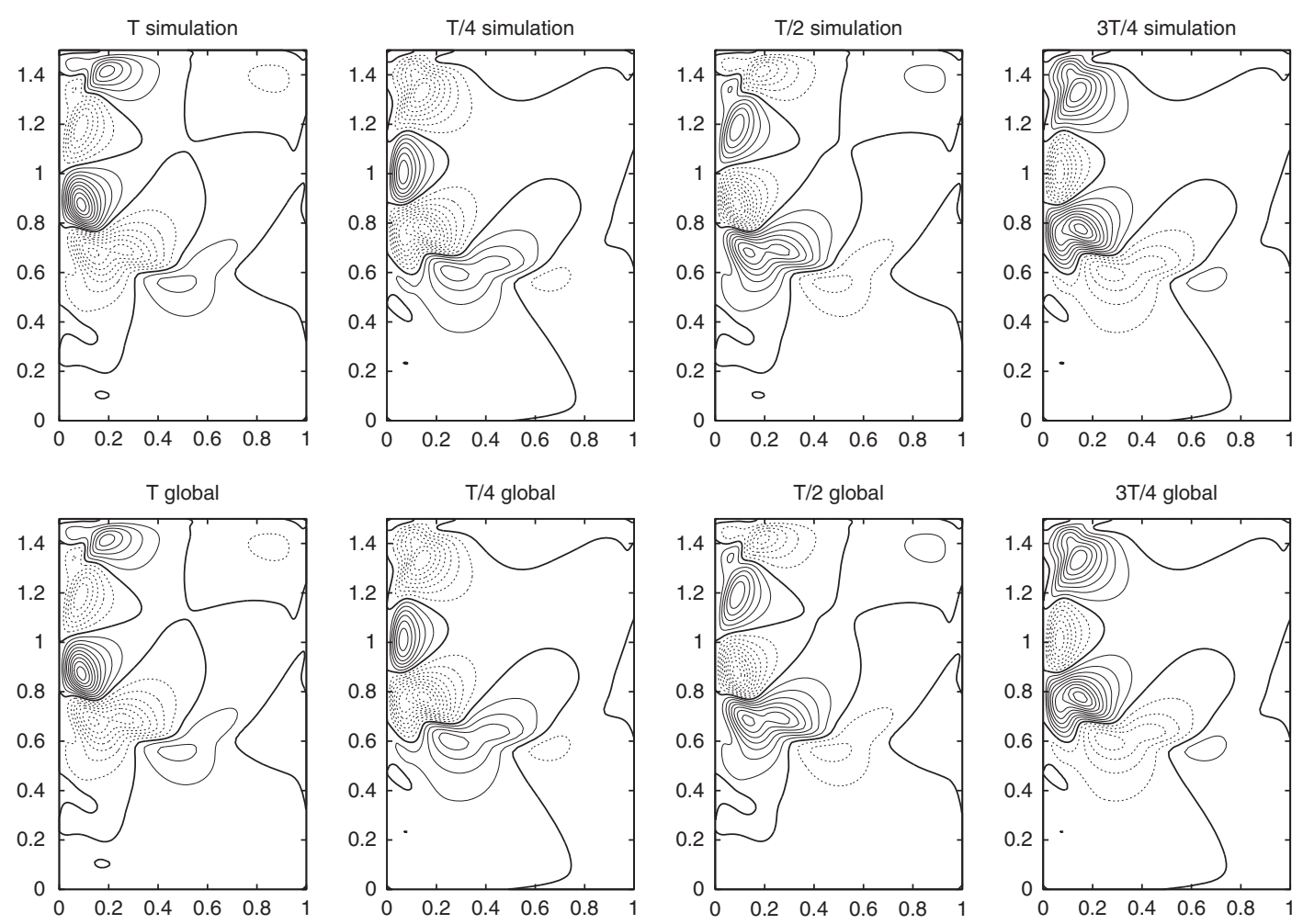

Figure 25. Comparisons of simulations (top row) and global analysis predictions (bottom row) for one period $T$ in intervals of $T / 4$ from left to right for aspect ratio $A=1.5$ and $\operatorname{Re}=5326.9$ with $M=501, N=141$. Contour levels are in intervals of 0.1 , with negative levels shown by dashed lines.

and Pierre [20]. Similarly $\Im\left(\lambda_{c}\right)$ differs by $22 \%$ with former and $31.3 \%$ with the latter. This significant difference of our results with the literature may be attributed to the limited grid checks done by these researchers as they used a single mesh size to determine the critical values. Figure 10(a) shows the difference in the values of $R e_{\mathrm{c}}$ for the coarse and fine grid sizes used in our study. This suggests that rigorous grid checks have to be carried out to determine the critical parameters.

Table VI shows the limited work done even in the case of $A=2.0$. We observe that the critical values differ significantly. In terms of $\%$ of relative difference, our $R e_{\mathrm{c}}$ value differ by $17 \%$ with Goodrich et al. [14] and 2.6\% with Abouhamza and Pierre [20], whereas $\Im\left(\lambda_{\mathrm{c}}\right)$ differ by $40 \%$ with the former and $22.3 \%$ with the latter. Goodrich et al. performed the numerical simulation on an impulsively started LDC at $R e=5000$ for two different grid sizes to find the Hopf bifurcation. They found the asymptotic periodic state with period $2.469 \leqslant T \leqslant 2.484\left(T=2 \pi / \Im\left(\lambda_{\mathrm{c}}\right)\right)$ on a grid size of $48 \times 96$ and $2.305 \leqslant T \leqslant 2.313$ on a $96 \times 192$ grid. Abouhamza and Pierre obtained the $R e_{\mathrm{c}}$ value as 5567 when the number of elements were 3136 (d.o.f=12 546) and 5715 when 3600 elements were used (d.o.f. $=14402$ ). This clearly shows that insufficient grid checks were done to predict the value of $R e_{\mathrm{c}}$ accurately. Though the values of the time period for the number of elements 

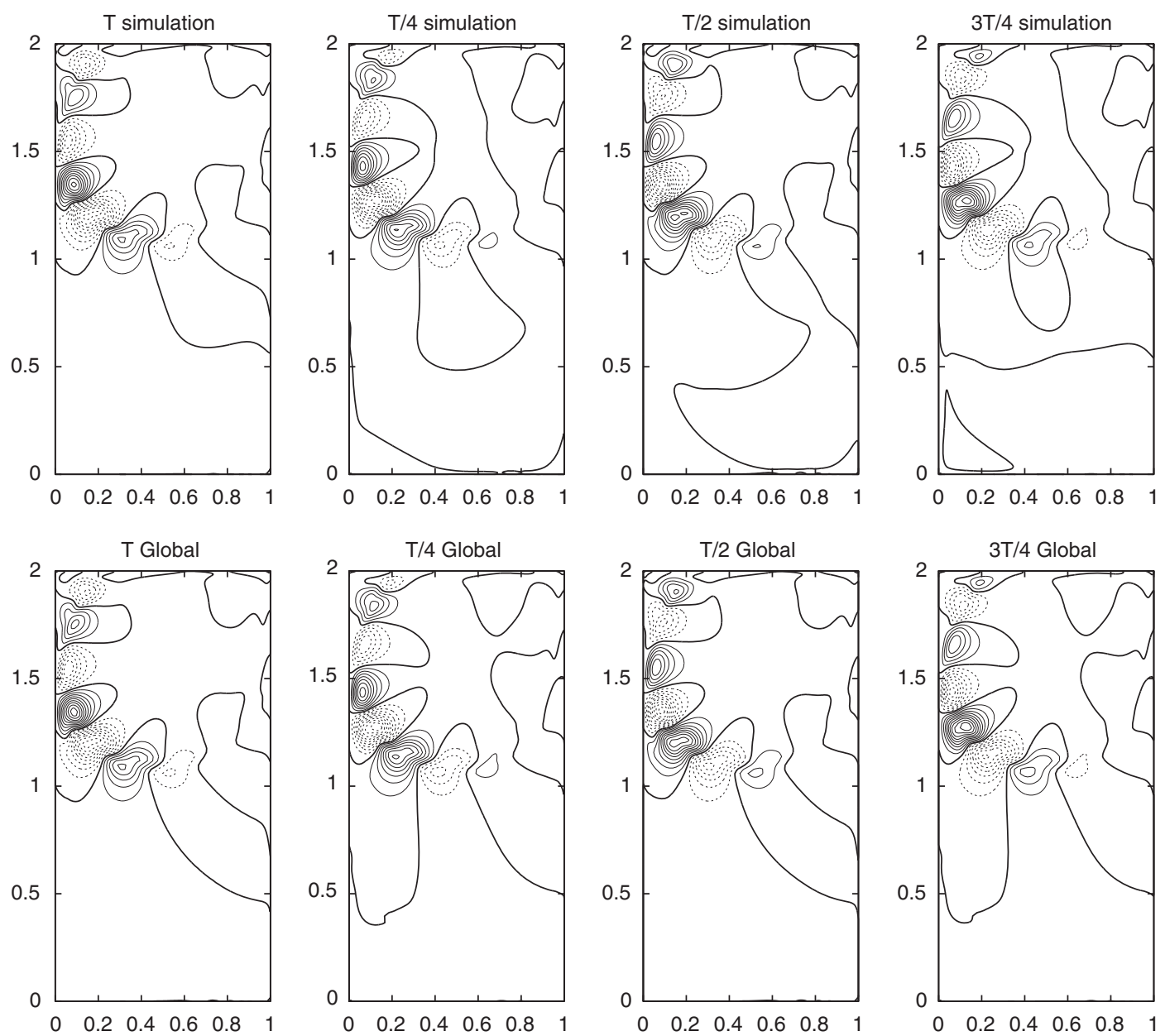

Figure 26. Comparisons of simulations (top row) and global analysis predictions (bottom row) for one period $T$ in intervals of $T / 4$ from left to right for aspect ratio $A=2$ and $R e=5861$ with $M=501, N=161$. Contour levels are in intervals of 0.1 , with negative levels shown by dashed lines.

mentioned above were found to be 2.023 and 2.015 and are in good agreement with Goodrich et al., the accuracy of these values is again under question as they were obtained for only two different grid sizes. Our studies also show that for $A=2$, unless a large number of eigenvalues are computed the predicted frequencies from the global analysis can be inaccurate. Moreover, our study emphasizes the importance of conducting independent simulations to confirm the predictions from a global stability analysis.

The critical values being different for each of the values of the aspect ratio in this table can be due to one of the following factors: grid checks, handling corner singularities, accurate computation of base flow. In our study, we made the best efforts in meeting these requirements that led us to calculate the critical values accurately for the different aspect ratios studied. 
GLOBAL FLOW INSTABILITY IN AN LDC

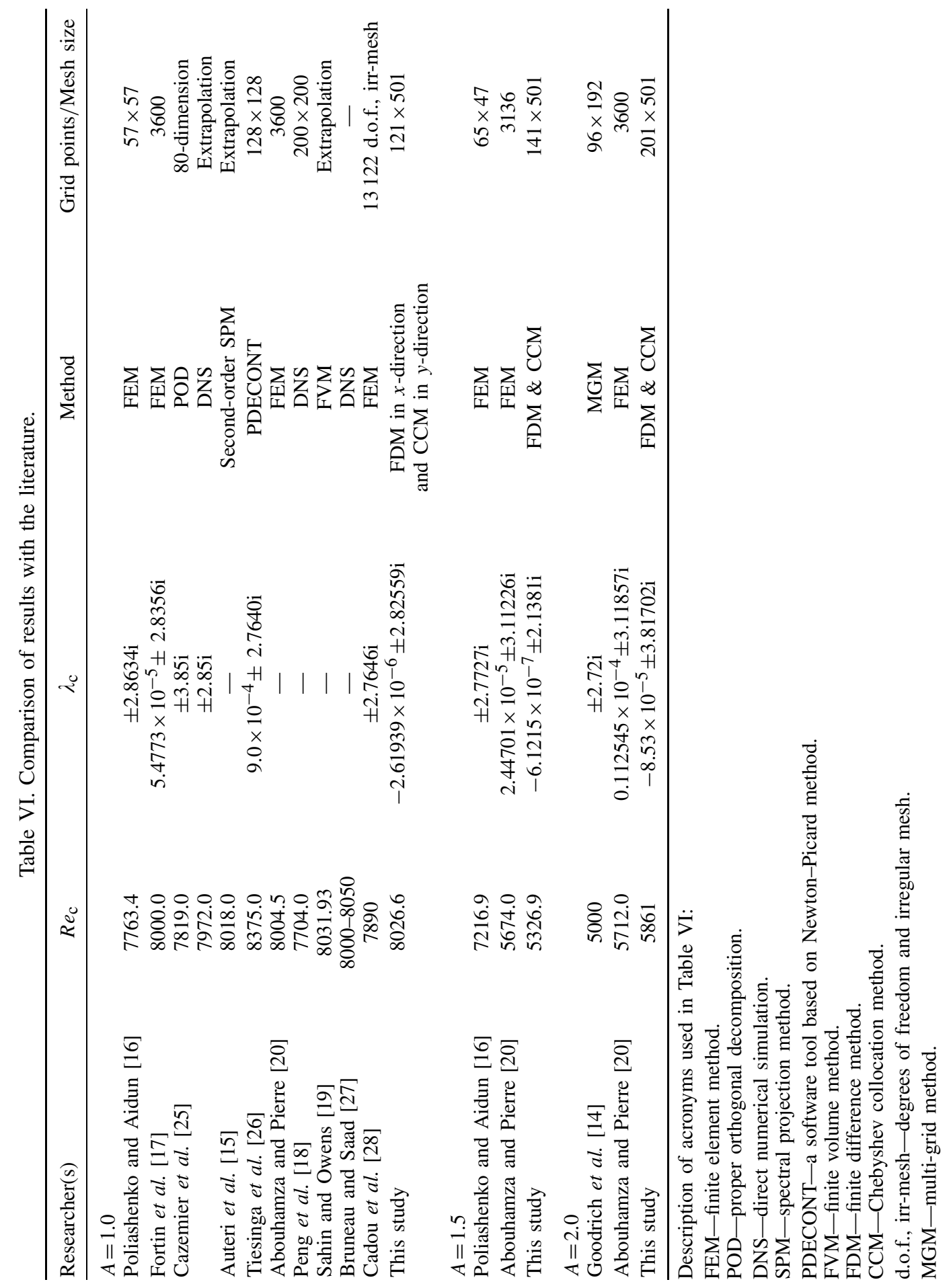

Copyright (C) 2009 John Wiley \& Sons, Ltd.

Int. J. Numer. Meth. Fluids (2009)

DOI: $10.1002 /$ fld 


\section{B. L. BOPPANA AND J. S. B. GAJJAR}

\section{CONCLUSIONS}

A new numerical technique is used to analyze the stability of a two-dimensional, unsteady and incompressible flow in the LDC of $A=1.0,1.5$ and 2.0. It is found in all of these configurations that flow loses stability to a Hopf bifurcation. We observe from our numerical experiments that $\Im\left(\lambda_{\mathrm{c}}\right)$ is less sensitive with grid size when compared with $R e_{\mathrm{c}}$. Extrapolation of the obtained numerical data estimated $R e_{\mathrm{c}}$ to be 8020.1 for $A=1.0, R e_{\mathrm{c}}=5332.1$ for $A=1.5$ and $R e_{\mathrm{c}}=5790$ for $A=2.0$. Though the values of $R e_{\mathrm{c}}$ do not agree with the literature for $A=1.5$ and 2.0, it is found to be reasonably consistent with those reported in the literature for $A=1.0$ and is in excellent agreement with our simulations of the linearized Navier-Stokes equations for all the aspect ratios studied.

The authors would like to thank the referees for their helpful comments.

\section{REFERENCES}

1. Burgraff OR. Analytical and numerical study of the structure of steady separated flows. Journal of Fluid Mechanics 1966; 24:113-151.

2. Ghia U, Ghia KN, Shin CT. High-Re solutions for incompressible flow using the Navier-Stokes equations and a multigrid method. Journal of Computational Physics 1982; 48:387-411.

3. Schreiber R, Keller HB. Driven cavity flows by efficient numerical techniques. Journal of Computational Physics $1983 ; 49: 310-333$.

4. Botella O, Peyret R. Benchmark spectral results on the lid-driven cavity flow. Computers and Fluids 1998; 24:421-433.

5. Auteri F, Quartapelle L, Vigevano L. Accurate $\omega-\psi$ spectra; solution of the singular driven cavity problem. Journal of Computational Physics 2002; 180:597-615.

6. Gajjar JSB, Azzam N. Solution of the steady Navier-Stokes equations for high Reynolds flows. Recent Res. Devel. Fluid Dynamics 2003; 4:181-199.

7. Azzam N. Numerical solution of the Navier-Stokes equations for the flow in a lid-driven cavity and a cylinder cascade. Ph.D. Thesis, University of Manchester, 2003.

8. Erturk E, Corke TC, Gökcöl C. Numerical solutions of 2-D steady incompressible driven cavity flow at high Reynolds numbers. International Journal for Numerical Methods in Fluids 2005; 48(7):747-774.

9. Batchelor GK. On steady laminar flow with closed streamlines at large Reynolds numbers. Journal of Fluid Dynamics 1956; 1:177-190.

10. Gajjar JSB, Azzam N. Numerical solution of the Navier-Stokes equations for the flow in a cylinder cascade. Journal of Fluid Dynamics 2004; 520:51-82.

11. Korolev G, Gajjar JSB, Ruban AI. Once again on the supersonic flow past a corner ramp. Journal of Fluid Dynamics 2002; 463:173-199.

12. Gustafson K, Halasi K. Vortex dynamics of cavity flows. Journal of Computational Physics 1986; 64:279-319.

13. Gustafson K, Halasi K. Cavity flow dynamics at higher Reynolds numbers and higher aspect ratio. Journal of Computational Physics 1987; 70:271-283.

14. Goodrich JW, Gustafson K, Halasi K. Hopf bifuraction in the driven cavity. Journal of Computational Physics 1990; 90:219-261.

15. Auteri F, Parolini N, Quartapelle L. Numerical investigation on the stability of singular driven cavity flow. Journal of Computational Physics 2002; 183:1-25.

16. Poliashenko M, Aidun CK. A direct method for computation of simple bifurcations. Journal of Computational Physics 1995; 121(2):246-260.

17. Fortin A, Jardak M, Gervais JJ, Pierre R. Localization of Hopf bifurcations in fluid flow problems. International Journal for Numerical Methods in Fluids 1997; 24(11):1185-1210.

18. Peng Y-F, Shiau Y-H, Hwang RR. Transition in a 2-D lid-driven cavity flow. Computers and Fluids 2003; 32:337-352.

19. Sahin M, Owens RG. A novel fully-implicit finite volume method applied to the lid-driven cavity problem. Part II. Linear stability analysis. International Journal for Numerical Methods in Fluids 2003; 42:79-88. 


\section{GLOBAL FLOW INSTABILITY IN AN LDC}

20. Abouhamza A, Pierre R. A neutral stability curve for incompressible flows in a rectangular driven cavity. Mathematical and Computer Modelling 2003; 38:141-157.

21. Canuto C, Hussaini AQMY, Zang T. Spectral Methods in Fluid Dynamics. Springer Series in Computational Physics. Springer: Berlin, 1987.

22. Lehoucq RB, Sorensen DC, Yang C. ARPACK User's Guide, Solution of Large-scale Eigenvalue Problems with Implicitly Restarted Arnoldi Methods. SIAM: Philadelphia, PA, 1998.

23. Bjørstad PE, Trøjstheim BP. High precision solutions of two fourth-order eigenvalue problems. Computing (Vienna/New York) 1999; 63(2):97-107.

24. Seydel R. Practical Bifurcation and Stability Analysis from Equilibrium to Chaos. Springer: Berlin, 1994.

25. Cazemier W, Verstappen RWCP, Veldman AEP. Proper orthogonal decomposition and low-dimensional models for driven cavity flows. Physics of Fluids 1998; 10(7):1685-1699.

26. Tiesinga G, Wubs FW, Veldman AEP. Bifurcation analysis of incompressible flow in a driven cavity by the Newton-Picard method. Journal of Computational and Applied Mathematics 2002; 140:751-772.

27. Bruneau CH, Saad M. The 2D lid-driven cavity problem revisited. Computers and Fluids 2006; 35(3):326-348.

28. Cadou JM, Potier-Ferry M, Cochelin B. A numerical method for the computation of bifurcation points in fluid mechanics. European Journal of Mechanics Fluids—B 2006; 25:234-254. 